\title{
BILIPSCHITZ EMBEDDING OF SELF-SIMILAR SETS
}

\author{
JUAN DENG, ZHI-YING WEN, YING XIONG, AND LI-FENG XI
}

\begin{abstract}
This paper proves that the self-similar set satisfying the strong separation condition can be bilipschitz embedded into self-similar set with larger Hausdorff dimension, and it can be embedded into a self-similar set with the same Hausdorff dimension if and only if these two self-similar sets are bilipschitz equivalent.
\end{abstract}

\section{INTRODUCTION}

For metric spaces $\left(A, d_{A}\right)$ and $\left(B, d_{B}\right)$, a bijection $f:\left(A, d_{A}\right) \rightarrow\left(B, d_{B}\right)$ is said to be bilipschitz, if there is a bilipschitz constant $C>0$ such that for all $x, y \in A$,

$$
C^{-1} d_{A}(x, y) \leq d_{B}(f(x), f(y)) \leq C d_{A}(x, y) .
$$

We say that $\left(A, d_{A}\right)$ and $\left(B, d_{B}\right)$ are bilipschitz equivalent, if there is a bilipschitz bijection from $\left(A, d_{A}\right)$ to $\left(B, d_{B}\right)$. We say that $\left(A, d_{A}\right)$ can be bilipschitz embedded into $\left(B, d_{B}\right)$, if there is a subset $B_{1}$ of $B$ such that $\left(A, d_{A}\right)$ and $\left(B_{1}, d_{B}\right)$ are bilipschitz equivalent.

As in [6], "topology" may be regarded as the study of equivalence classes of sets under homeomorphism, and "fractal geometry" is sometimes thought of as the study of equivalence classes of fractals under bilipschitz mappings. Another interesting motivation of studying bilipschitz equivalence of fractals comes from geometry group theory $([1],[7])$.

Many works have been devoted to the related topics. For example, Cooper and Pignataro [2], Falconer and Marsh [5, 6], David and Semmes [3] and Xi [19, 20] studied the shape of Cantor set, nearly Lipschitz equivalence, BPI equivalence and quasi-Lipschitz equivalence respectively. Xi et al. ([15]-[16], [21]-[25]) also discussed the bilipschitz equivalence between self-similar sets.

Example 1. For two self-similar sets with the same dimension, they may be not bilipschitz equivalent. For example, let $3 r^{\log 2 / \log 3}=1$, suppose a self-similar set is generated by similitudes $r x, r x+(1-r) / 2$ and $r x+1-r$, then this self-similar set and the Cantor ternary set have the same Hausdorff dimension $\log 2 / \log 3$, but they are not bilipschitz equivalent $([6])$.

On the bilipschitz equivalence of self-similar sets, the following results are known.

1) Self-similar sets satisfying the strong separation condition (SSC in short). Falconer and Marsh [6] gave a necessary condition such that they are bilipschitz equivalent. A necessary and sufficient condition was also obtained by $\mathrm{Xi}[21]$.

2000 Mathematics Subject Classification. 28A80.

Key words and phrases. fractal, self-similar set, bilipschitz embedding, bilipschitz equivalence.

This work is supported by National Natural Science Foundation of China (Grant Nos 10671180,10571140, 10571063,10631040, 11071164) and Morningside Center of Mathematics. 
2) Self-similar sets satisfying open set condition (OSC in short).

Define two self-similar sets respectively by $F_{i}=F_{i} / 5 \cup\left((i+1) / 5+F_{i} / 5\right) \cup(4 / 5+$ $\left.F_{i} / 5\right)(i=1,2)$. Then both $F_{1}$ and $F_{2}$ satisfy OSC and have the same Hausdorff dimension. David and Semmes [3] asked whether they are bilipschitz equivalence, and the question is called " $\{1,3,5\}-\{1,4,5\}$ problem."

Rao, Ruan and Xi [15] gave an affirmative answer to the problem, then Xi et al $[16,22,23]$ studied some generalizations, recently Xi and Xiong [25] dealt with the problem in high dimension.

3) Connected self-similar sets.

Wen and Xi [18] discussed the geometric condition for self-similar arcs to be bilipschitz equivalent. They also constructed two self-similar $\operatorname{arcs} \gamma_{1}, \gamma_{2}$ with the same Hausdorff dimension but $\gamma_{1}$ and $\gamma_{2}$ are not bilipschitz equivalent.

4) Bilipschitz embedding between regular set and self-similar set.

Mattila and Saaranen [12] discussed the bilipschitz embedding for Ahlfors regular sets, and proved that for any $t$-regular set $F$ with $t>s$ that there exists a self-similar set $E$, generated by similitudes of the same ratio, such that $E$ can be bilipschitz embedded into $F$.

Here we recall the notion of $s$-regular set.

Definition 1. Let $E \subset X$ and $s>0$. We say that $E$ is $s$-regular, if $E$ is closed and if there exists a Borel measure $\mu$ on $X$ and a constant $C_{E} \geq 1$ such that $\mu(X \backslash E)=0$ and $r^{s} \leq \mu(B(x, r)) \leq C_{E} r^{s}$ for all $x \in E, 0<r \leq|E|$ and $r<\infty$, where $B(x, r)$ is the closed ball centered at $x$ with radius $r$.

Remark 1. A self-similar set with OSC of Hausdorff dimension $t$ is $t$-regular ([9]). But if the self-similar set without OSC, the conclusion may be false. For example, let $\mathrm{E}_{\lambda}$ be self-similar set generalized by $S_{1}(x)=x / 3, S_{\lambda}(x)=x / 3+\lambda$ and $S_{3}(x)=$ $x / 3+2 / 3$, then by [10], $\operatorname{dim}_{H} E_{\lambda}=1$ and $\mathcal{H}^{1}\left(E_{\lambda}\right)=0$ for some $\lambda$, and $E_{\lambda}$ is not 1-regular in this case.

Recall that a self-similar set $E=\cup_{i=1}^{m} S_{i}(E)$ satisfies the strong separation condition (SSC), if $S_{i}(E) \cap S_{j}(E)=\varnothing$ for any $i \neq j$.

We will state our results as follows.

Theorem 1. Suppose $E_{1}$ and $E_{2}$ are self-similar sets with $\operatorname{dim}_{H} E_{1}<\operatorname{dim}_{H} E_{2}$. If $E_{1}$ satisfies the strong separation condition, then there is a bilipschitz map $g$ : $E_{1} \rightarrow g\left(E_{1}\right) \subset E_{2}$.

Remark 2. We stress that in this theorem, the condition $O S C$ is not required for $E_{2}$.

Theorem 2. Suppose $F$ and $F^{\prime}$ are self-similar sets satisfying the strong separation condition, and $\operatorname{dim}_{H} F=\operatorname{dim}_{H} F^{\prime}=s$. Then there is a bilipschitz map $h: F \rightarrow$ $h(F) \subset F^{\prime}$ if and only if $F$ and $F^{\prime}$ are bilipschitz equivalent.

In fact, Theorem 2 is the consequence of the following stronger result.

Theorem 3. Preserve the assumption in Theorem 2. If there are $K \subset F, K^{\prime} \subset F^{\prime}$ such that $K$ and $K^{\prime}$ are bilipschitz equivalent with $\mathcal{H}^{s}(K), \mathcal{H}^{s}\left(K^{\prime}\right)>0$, then $F$ and $F^{\prime}$ are bilipschitz equivalent.

Remark 3. David and Semmes introduced the BPI (big pieces of itself) equivalence, which is a few weaker than bilipschitz equivalence, and discussed some properties [3]. In particular, they proved that self-similar sets $F$ and $F^{\prime}$ with OSC are 
BPI equivalent, if and only if there are $K \subset F, K^{\prime} \subset F^{\prime}$ such that $K$ and $K^{\prime}$ are bilipschitz equivalent with $\mathcal{H}^{s}(K), \mathcal{H}^{s}\left(K^{\prime}\right)>0$ (Proposition 7.1 of [3]). Then Theorem 3 shows that self-similar sets satisfying SSC are bilipschitz equivalent if and only if they are BPI equivalent. Here is an open problem: under what condition, BPI equivalence implies bilipschitz equivalence for self-similar sets.

Given a metric space $(X, d)$, let $\left|X_{1}\right|$ denote the diameter of set $X_{1}(\subset X)$, and $d\left(X_{1}, X_{2}\right)$ is the distance between subsets $X_{1}$ and $X_{2}$ of $X$.

Definition 2. Let $C, \delta, s$ be positive numbers. A sequence $\left\{\Phi_{k}\right\}_{k \geq 0}$ consisting of finite index is called controlled by $(C, \delta, s)$ provided

(a) For any $k \geq 1, \Phi_{k}$ is a collection of words with length $k$, by convention, $\Phi_{0}=\{\emptyset\}$, where $\emptyset$ is the empty word;

(b) If $i_{1} \cdots i_{k-1} i_{k} \in \Phi_{k}$, then $i_{1} \cdots i_{k-1} \in \Phi_{k-1}$;

(c) For any $k_{2}>k_{1} \geq 0$ and any $i_{1} i_{2} \cdots i_{k_{1}} \in \Phi_{k_{1}}$,

$$
\operatorname{card}\left\{i_{1} i_{2} \cdots i_{k_{1}} \cdots i_{k_{2}} \in \Phi_{k_{2}}\right) \leq C\left(\delta^{-s}\right)^{k_{2}-k_{1}} .
$$

Definition 3. Let $(X, d)$ be a metric space, $C>1,0<\delta<1, s>0$, and let $\left\{\Phi_{k}\right\}_{k \geq 1}$ be a sequence controlled by $(C, \delta, s)$. Suppose $E \subset X$ and for any $k \in \mathbb{N}$, there is a decomposition of $E$ with respect to the sequence $\left\{\Phi_{k}\right\}_{k \geq 0}$ :

$$
E=\bigcup_{i_{1} i_{2} \cdots i_{k} \in \Phi_{k}} E^{i_{1} i_{2} \cdots i_{k}}
$$

We say that the set $E$ has $s$-structure if for any $i_{1} i_{2} \cdots i_{k} \in \Phi_{k}$, we have

1) $E^{i_{1} i_{2} \cdots i_{k}}=\bigcup_{i_{1} i_{2} \cdots i_{k} j \in \Phi_{k+1}} E^{i_{1} i_{2} \cdots i_{k} j}$

2) $\left|E^{i_{1} i_{2} \cdots i_{k}}\right| \leq C \delta^{k}$

3) $d\left(E^{i_{1} i_{2} \cdots i_{k}}, E^{j_{1} j_{2} \cdots j_{k}}\right) \geq C^{-1} \delta^{k}$ whenever $i_{1} \cdots i_{k} \neq j_{1} \cdots j_{k}$.

The following proposition shows that many typical fractals, including self-similar sets with SSC, have $s$-structure. Some self-similar sets with OSC (but without SSC) can be regarded as graph-directed sets or homogeneous Moran sets, and thus also have $s$-structure as shown below.

Proposition 1. Let $s>0$, then the following sets have $s$-structure:

(1) Bounded $s$-regular set with $s \in(0,1)$;

(2) $C^{1+\alpha}(\alpha>0)$ self-conformal set with dimension $s$ (in particular self-similar set satisfying $\mathrm{SSC}$;

(3) Graph-directed sets (on a transitive graph) satisfying SSC with dimension $s$;

(4) Homogeneous Moran set in $\mathcal{M}(J, \bar{n}, \bar{c})(\bar{n} \bar{c}<1)$ with dimension $s$.

The fractal classes (2)-(4) will be introduced in Section 3.

Remark 4. In Section 6, we will prove that: let $E \subset[0,1]$, if $E$ has positive Lebesgue measure, then $E$ has no $s$-structure for any $s$.

Proposition 2. If $E \subset X$ has $s$-structure and $F \subset Y$ is $t$-regular with $s<t$, then there is a bilipschitz map $f: E \rightarrow f(E) \subset F$.

The above proposition establishes the bilipschitz embedding from a set having $s$-structure to a regular set. Notice that $s$-dimensional self-similar sets with SSC have $s$-structure, this proposition is the complementarity of the result of Mattila and Saaranen mentioned above [12]. It is also proved in [12] that any $s$-regular set 
with $s \in(0,1)$ can be embedded into any $t$-regular set $F$ with $t>s$. This result is exactly a consequence of Proposition 2 and Proposition 1.(1).

As shown in Remark 1 , self-similar set of dimension $t$ maybe not $t$-regular, however the following proposition shows that it can contain a $t^{\prime}$-regular subset with $t^{\prime}(<t)$ close to $t$.

Proposition 3. If $F$ is a self-similar set with $\operatorname{dim}_{H} F=t$. For any $\varepsilon>0$, there is a self-similar set $F_{\varepsilon}$ satisfying the strong separation condition such that $F_{\varepsilon} \subset F$ and $\operatorname{dim}_{H} F_{\varepsilon} \in(t-\varepsilon, t]$. Here $F_{\varepsilon}$ is $t_{\varepsilon}$-regular with $t_{\varepsilon} \in(t-\varepsilon, t]$.

The paper is organized as follows. Sections 2, 3 and 4 are devoted mainly to the proofs of Propositions 1, 2 and 3 respectively. Then Theorem 1 follows from Proposition 1.(2), Proposition 2 and Proposition 3. In Section 5, we prove Theorem 3 on the technique of [21]. In the last section, we show that any subset of [0,1] with positive Lebesgue measure cannot be embedded into any self-similar set with SSC.

\section{Proof of Proposition 1}

\subsection{Finite Words.}

Given integer $n \geq 2$, let $\Sigma_{n}^{*}$ be the collection of finite words composed of $1, \cdots, n$, that means

$$
\begin{aligned}
\Sigma_{n}^{*} & =\cup_{k \geq 0}\{1, \cdots, n\}^{k} \\
& =\{\emptyset\} \cup\left\{j_{1} \cdots j_{k}: k \geq 1 \text { and } j_{t} \in \mathbb{N} \cap[1, n] \text { for } 1 \leq t \leq k\right\},
\end{aligned}
$$

where $\emptyset$ is the empty word.

For word $\mathbf{i}=i_{1} \cdots i_{k}$ and $\mathbf{j}=j_{1} \cdots j_{l}$, set $\mathbf{i} * \mathbf{j}=i_{1} \cdots i_{k} j_{1} \cdots j_{l}$. For word $\mathbf{i}=i_{1} \cdots i_{k}$, its length $|\mathbf{i}|$ is defined to be $k$.

\subsection{Self-conformal Set.}

We say that a mapping $f: U\left(\subset \mathbb{R}^{l}\right) \rightarrow \mathbb{R}^{l}$ is $C^{1+\alpha}$ conformal with $\alpha>0$, if $U$ is open, $D f(x)$ is a contracting similarity for any $x \in U$, and there is a constant $C_{f}$ such that for all $x, y \in U$,

$$
|D f(x)-D f(y)| \leq C_{f}|x-y|^{\alpha} .
$$

We say that a set $E$ is a $C^{1+\alpha}$ self-conformal set, if there are $C^{1+\alpha}$ contracting and conformal mappings $\left\{f_{1}, \cdots, f_{m}\right\}$ such that

$$
E=\cup_{i=1}^{m} f_{i}(E)
$$

is a disjoint union, that is $f_{i}(E) \cap f_{j}(E)=\varnothing$ whenever $i \neq j$.

Notice that for $C^{1+\alpha}$ self-conformal set $E$ of Hausdorff dimension $s$,

$$
0<\mathcal{H}^{s}(E)<\infty
$$

In [6], [19] and [20], it is proved that $C^{1+\alpha}$ self-conformal sets with the same Hausdorff dimension are both nearly Lipschitz equivalent and quasi-Lipschitz equivalent. 


\subsection{Graph-directed Sets.}

Suppose $G$ is a directed graph, which contains $n$ vertexes $\{1, \cdots, n\}$ and directed edges $\{e: e \in G\}$ among these vertexes. For each edge $e$, there is a contracting similitude $S_{e}: \mathbb{R}^{l} \rightarrow \mathbb{R}^{l}$. Let $\mathcal{E}_{i, j}$ be the set of all the edges from vertex $i$ to vertex $j$. By [14] there is a family $\left\{K_{1}, \cdots, K_{n}\right\}$ of compact sets in $\mathbb{R}^{l}$ such that

$$
K_{i}=\bigcup_{j} \bigcup_{e \in \mathcal{E}_{i, j}} S_{e}\left(K_{j}\right)
$$

We say $\left\{K_{i}\right\}_{i}$ satisfies SSC, if the right hand of (2.1) is a disjoint union. The graph is said to be transitive, if for any vertexes $i, j$, there is a path starting at $i$ and ending at $j$. For graph-directed sets $\left\{K_{i}\right\}_{i}$ satisfying SSC on a transitive graph,

$$
0<\mathcal{H}^{s}\left(K_{i}\right)<\infty \text { with } s=\operatorname{dim}_{H}\left(K_{1}\right)=\cdots=\operatorname{dim}_{H}\left(K_{n}\right)
$$

\subsection{Homogeneous Moran Set.}

Given integer $\bar{n} \geq 2$ and $\bar{c}>0$ with $\bar{n} \bar{c}<1$, we recall some notions of homogeneous Moran class $\mathcal{M}(J, \bar{n}, \bar{c})$.

Definition 4. Suppose that $J \subset \mathbb{R}^{1}$ is a closed interval. For a collection $\mathcal{F}=$ $\left\{J_{\mathbf{i}}: \mathbf{i} \in \Sigma_{(\bar{n})}^{*}\right\}$ of closed subintervals of $J=J_{\emptyset}$, we say $\mathcal{F}$ has homogeneous Moran structure $(J, \bar{n}, \bar{c})$, if for any word $\mathbf{i}, J_{\mathbf{i} * 1}, J_{\mathbf{i} * 2}, \cdots, J_{\mathbf{i} * \bar{n}}$ are subsets of $J_{\mathbf{i}}$ such that

$$
\operatorname{int} J_{\mathbf{i} * i} \cap \operatorname{int} J_{\mathbf{i} * j}=\varnothing \text { whenever } i \neq j \text {, }
$$

and for each $j$,

$$
\left|J_{\mathbf{i} * j}\right| /\left|J_{\mathbf{i}}\right|=\bar{c} .
$$

A homogeneous Moran set determined by $\mathcal{F}$ is defined by

$$
E(\mathcal{F})=\bigcap_{k \geq 1} \bigcup_{|\mathbf{i}|=k} J_{\mathbf{i}}
$$

where any interval $J_{\mathbf{i}}$ in $\mathcal{F}$ is called a basic element of $E$. Let $\mathcal{M}(J, \bar{n}, \bar{c})$ be the collection of all the homogeneous Moran sets with structure $(J, \bar{n}, \bar{c})$.

Remark 5. By the definition above, we see that two Moran sets having the same Moran structure only differ from the relative positions of the basic elements of same order. In particular, the basic elements $J_{\mathbf{i} * i}, J_{\mathbf{i} * j}$ can share one endpoint.

Example 2. Let $\bar{n}=3$ and $\bar{c}=1 / 5$. Suppose $J=[0,1]$ and $\mathcal{F}=\left\{J_{\mathbf{i}}: \mathbf{i} \in \Sigma_{3}^{*}\right\}$ is given by

$$
J_{i_{1} \cdots i_{k}}=\left[\sum_{t=1}^{k} \theta\left(i_{t}\right) / 5^{t}, 1 / 5^{k}+\sum_{t=1}^{k} \theta\left(i_{t}\right) / 5^{t}\right]
$$

where $\theta(1)=0, \theta(2)=3, \theta(3)=4$. Then the corresponding homogeneous Moran set is called $\{1,4,5\}$-set. Here $J_{i_{1} \cdots i_{k} 2} \cap J_{i_{1} \cdots i_{k} 3} \neq \varnothing$.

In fact, for any $E \in \mathcal{M}(J, \bar{n}, \bar{c})$, we have $\operatorname{dim}_{H} E=-\log \bar{n} / \log \bar{c}$. For the results on the dimensions of Moran sets, we refer to [8, 13, 17].

\subsection{Proof of Proposition 1.}

(1) Bounded $s$-regular set $F$ with $s \in(0,1)$ :

Since $F(\subset X)$ is $s$-regular, there exists a Borel measure $\mu$, supported on $F$, such that for $x \in F, 0<r \leq|F|<\infty$,

$$
r^{s} \leq \mu(B(x, r) \cap F) \leq C_{F} r^{s}
$$

where the constant $C_{F}>0$. Let $D_{F}=\left(3 C_{F} 2^{s}\right)^{1 /(1-s)}+1$. 
Lemma 1. [12] Let $0<s<1, R>0$, let $E \subset X$ be closed and bounded and let $\mu_{0}$ be a Borel measure on $X$ such that $\mu_{0}(X \backslash E)=0$ and that $\mu_{0}(B(x, r)) \leq C_{F} r^{s}$ for all $x \in E, r>0$, and $\mu_{0}(B(x, r)) \geq r^{s}$ for all $x \in E, 0<r<R$. For every $0<r<R /\left(2 D_{F}\right)$ there exist disjoint closed balls $B\left(x_{i}, r\right), i=1, \cdots, m$, and positive numbers $\rho_{i}, r \leq \rho_{i} \leq\left(D_{F}\right) r$, such that $m \leq C_{F}|E|^{s} / r^{s}, x_{i} \in E$, $x_{j} \notin B\left(x_{i}, \rho_{i}\right)$ for $i<j$,

$$
E \subset \cup_{i=1}^{m} B\left(x_{i}, \rho_{i}\right) \text { and } E \cap\left[B\left(x_{i}, \rho_{i}+r\right) \backslash B\left(x_{i}, \rho_{i}\right)\right]=\varnothing .
$$

Fix $x^{*} \in F$ and take $\delta$ small enough.

Without loss generality, we assume that $|F| \leq 1=\delta^{0}$, then $F \subset B\left(x^{*}, \delta^{0}\right)$. By convention, $x_{\emptyset}=x^{*}$ and $C_{\emptyset}=1$, then we get $x_{i_{1} \cdots i_{k}}=x^{*}$ and $C_{i_{1} \cdots i_{k}}=1$ for $k=0$.

By induction on $k$, assume that we get closed balls

$$
B\left(x_{i_{1} \cdots i_{k}}, C_{i_{1} \cdots i_{k}} \delta^{k}\right) \text { with } 1 \leq C_{i_{1} \cdots i_{k}} \leq D_{F}
$$

and

$$
F \cap\left[B\left(x_{i_{1} \cdots i_{k}}, C_{i_{1} \cdots i_{k}} \delta^{k}+\delta^{k}\right) \backslash B\left(x_{i_{1} \cdots i_{k}}, C_{i_{1} \cdots i_{k}} \delta^{k}\right)\right]=\varnothing,
$$

where $i_{t} \leq\left[C_{F} 2^{s} D_{F}^{s}\right] \delta^{-s}$ for all $t$.

Let $\mu_{0}=\left.\mu\right|_{B\left(x_{i_{1} \cdots i_{k}}, C_{i_{1} \cdots i_{k}} \delta^{k}\right)}$, then $\mu_{0}=\left.\mu\right|_{B\left(x_{i_{1} \cdots i_{k}}, C_{i_{1} \cdots i_{k}} \delta^{k}+\delta^{k}\right)}$ by (2.4). Applying Lemma 1 to the case

$$
E=B\left(x_{i_{1} \cdots i_{k}}, C_{i_{1} \cdots i_{k}} \delta^{k}\right) \cap F, R=\delta^{k}, r=\delta^{k+1} \text { and } \mu_{0}=\left.\mu\right|_{B\left(x_{i_{1} \cdots i_{k}}, C_{i_{1} \cdots i_{k}} \delta^{k}\right)},
$$

we get $m_{i_{1} \cdots i_{k}}$ closed balls $\left\{B\left(x_{i_{1} \cdots i_{k} i_{k+1}}, C_{i_{1} \cdots i_{k} i_{k+1}} \delta^{k+1}\right)\right\}_{i_{k+1}=1}^{m_{i_{1} \cdots i_{k}}}$ such that

$$
B\left(x_{i_{1} \cdots i_{k}}, C_{i_{1} \cdots i_{k}} \delta^{k}\right) \cap F=\cup_{i_{k+1}=1}^{m_{i_{1} \cdots i_{k}}}\left[B\left(x_{i_{1} \cdots i_{k} i_{k+1}}, C_{i_{1} \cdots i_{k} i_{k+1}} \delta^{k+1}\right) \cap F\right],
$$

and for $i_{k+1} \neq i_{k+1}^{\prime}$,

$$
d\left(\left(B\left(x_{i_{1} \cdots i_{k} i_{k+1}}, C_{i_{1} \cdots i_{k} i_{k+1}} \delta^{k+1}\right), B\left(x_{i_{1} \cdots i_{k} i_{k+1}^{\prime}}, C_{i_{1} \cdots i_{k} i_{k+1}^{\prime}} \delta^{k+1}\right)\right) \geq \delta^{k+1},\right.
$$

where

$$
m_{i_{1} \cdots i_{k}} \leq C_{F}|E|^{s} / r^{s} \leq\left[C_{F} 2^{s} D_{F}^{s}\right] \delta^{-s} \text { and } C_{i_{1} \cdots i_{k} i_{k+1}} \leq D_{F} .
$$

Then the inductive assumptions (2.3) and (2.4) are true for every $k$.

Let $F^{i_{1} \cdots i_{k}}=B\left(x_{i_{1} \cdots i_{k}}, C_{i_{1} \cdots i_{k}} \delta^{k}\right) \cap F$, then

$$
\left|F^{i_{1} \cdots i_{k}}\right| \leq\left(2 D_{F}\right) \delta^{k}
$$

For any $F^{i_{1} \cdots i_{k_{1}}}$, we are going to estimate the cardinality of its subset in the form $F^{i_{1} \cdots i_{k_{1}} \cdots i_{k_{2}}}$. Since

by $(2.2)$, we have

$$
F^{i_{1} \cdots i_{k_{1}}}=\bigcup_{i_{1} \cdots i_{k_{1}} \cdots i_{k_{2}}} F^{i_{1} \cdots i_{k_{1}} \cdots i_{k_{2}}}
$$

$$
\mu\left(F^{i_{1} \cdots i_{k_{1}}}\right) \leq C_{F}\left(C_{i_{1} \cdots i_{k_{1}}} \delta^{k_{1}}\right)^{s} \leq\left(C_{F} D_{F}^{s}\right) \delta^{k_{1} s},
$$

and

$$
\mu\left(F^{i_{1} \cdots i_{k_{1}} \cdots i_{k_{2}}}\right) \geq \delta^{k_{2} s} .
$$

Therefore, for $i_{1} \cdots i_{k_{1}}$ fixed, we have

$$
\operatorname{card}\left\{i_{1} \cdots i_{k_{1}} i_{k_{1}+1} \cdots i_{k_{2}}\right\} \leq\left(C_{F} D_{F}^{s}\right)\left(\delta^{-s}\right)^{k_{2}-k_{1}} .
$$

It follows from (2.5)-(2.8) that $F$ has $s$-structure.

(2) $C^{1+\alpha}(\alpha>0)$ self-conformal set (in particular, self-similar set satisfying SSC):

Let $E$ be the invariant of the contracting conformal mappings $\left\{f_{1}, \ldots, f_{n}\right\}$ with $\operatorname{dim}_{H} E=s$. We will show that $E$ has $s$-structure. 
Write $f_{i_{1} \cdots i_{m}}=f_{i_{1}} \circ \cdots \circ f_{i_{m}}$ and $E_{i_{1} i_{2} \ldots i_{m}}=f_{i_{1} \cdots i_{m}}(E), 1 \leq i_{j} \leq n(j=$ $1, \cdots, m)$.

Fix a point $x \in E$. By [4], it is known that there is constant $\lambda>0$ such that for any $i_{1} i_{2} \cdots i_{m}$ and any $y \in E$,

$$
\begin{aligned}
\lambda^{-1} & \leq\left|E_{i_{1} i_{2} \cdots i_{m}}\right| /\left|f_{i_{1} i_{2} \cdots i_{m}}^{\prime}(x)\right| \leq \lambda, \\
\lambda^{-1} & \leq \mathcal{H}^{s}\left(E_{i_{1} i_{2} \ldots i_{m}}\right) /\left|f_{i_{1} i_{2} \ldots i_{m}}^{\prime}(x)\right|^{s} \leq \lambda, \\
\lambda^{-1} & \leq\left|f_{i_{1} i_{2} \cdots i_{m}}^{\prime}(y)\right| /\left|f_{i_{1} i_{2} \cdots i_{m}}^{\prime}(x)\right| \leq \lambda .
\end{aligned}
$$

Taking $\delta$ small enough, for any infinite sequence $i_{1} i_{2} \cdots i_{m} \cdots$ and any $k \geq 1$, we can choose the least integer $l(k)$ such that $\left|f_{i_{1} i_{2} \cdots i_{l(k)}}^{\prime}(x)\right|<\delta^{k}$, then $\left|f_{i_{1} \cdots i_{l(k)-1}}^{\prime}(x)\right| \geq \delta^{k}$, and

$$
\begin{aligned}
& \left|f_{i_{1} i_{2} \cdots i_{l(k)}}^{\prime}(x)\right| /\left|f_{i_{1} i_{2} \cdots i_{l(k)-1}}^{\prime}(x)\right| \\
= & \left|f_{i_{l(k)}}^{\prime}(x)\right| \cdot\left(\left|f_{i_{1} i_{2} \cdots i_{l(k)-1}}^{\prime}\left(f_{i_{l(k)}}(x)\right)\right| /\left|f_{i_{1} i_{2} \cdots i_{l(k)-1}}^{\prime}(x)\right|\right) \\
\geq & \left(\min _{1 \leq i \leq n} \inf _{z}\left|f_{i}^{\prime}(z)\right|\right) \lambda^{-1} .
\end{aligned}
$$

Therefore,

$$
\left(\min _{1 \leq i \leq n} \inf _{z}\left|f_{i}^{\prime}(z)\right|\right) \lambda^{-1} \delta^{k} \leq\left|f_{i_{1} i_{2} \cdots i_{l(k)}}^{\prime}(x)\right|<\delta^{k} .
$$

Let $\Pi_{k}$ be the collection of all the words $i_{1} i_{2} \ldots i_{l(k)}$ defined above. Then

$$
E=\cup_{i_{1} \cdots i_{p} \in \Pi_{k}} E_{i_{1} \cdots i_{p}} .
$$

The strong separation condition implies that there is a constant $C_{1}>0$ such that

$$
d\left(E_{i_{1} i_{2} \ldots i_{p}}, E \backslash E_{i_{1} i_{2} \ldots i_{p}}\right) \geq C_{1}\left|f_{i_{1} i_{2} \cdots i_{p}}^{\prime}(x)\right| \text { for all } i_{1} \cdots i_{p} .
$$

Then for any $i_{1} i_{2} \cdots i_{p} \in \Pi_{k}$, we have

$$
E_{i_{1} \cdots i_{p}}=\cup_{i_{1} \cdots i_{p} \cdots i_{q} \in \Pi_{k+1}} E_{i_{1} \cdots i_{p} \cdots i_{q}},
$$

and

$$
\begin{gathered}
\left|E_{i_{1} \cdots i_{p}}\right| \leq \lambda\left|f_{i_{1} i_{2} \cdots i_{p}}^{\prime}(x)\right|<\lambda \delta^{k} . \\
d\left(E_{i_{1} i_{2} \ldots i_{p}}, E \backslash E_{i_{1} i_{2} \ldots i_{p}}\right) \geq\left[C_{1}\left(\min _{1 \leq i \leq n} \inf _{z}\left|f_{i}^{\prime}(z)\right|\right) \lambda^{-1}\right] \delta^{k} .
\end{gathered}
$$

Fix any $i_{1} i_{2} \cdots i_{p} \in \Pi_{k_{1}}$ and $k_{1}<k_{2}$, we estimate the cardinality of the set

$$
\left\{i_{1} \cdots i_{p} \cdots i_{q}: i_{1} \cdots i_{p} \cdots i_{q} \in \Pi_{k_{2}}\right\} \text {. }
$$

In fact,

where

$$
\mathcal{H}^{s}\left(E_{i_{1} \cdots i_{p}}\right)=\sum_{i_{1} \cdots i_{p} \cdots i_{q} \in \Pi_{k_{2}}} \mathcal{H}^{s}\left(E_{i_{1} \cdots i_{p} \cdots i_{q}}\right)
$$

$$
\mathcal{H}^{s}\left(E_{i_{1} \cdots i_{p}}\right) \leq \lambda\left|f_{i_{1} i_{2} \cdots i_{p}}^{\prime}(x)\right|^{s} \leq \lambda \delta^{k_{1} s},
$$

and by (2.9),

$$
\begin{aligned}
\mathcal{H}^{s}\left(E_{i_{1} \cdots i_{p} \cdots i_{q}}\right) & \geq\left(\lambda^{-1}\right)\left|f_{i_{1} i_{2} \cdots i_{p} \cdots i_{q}}^{\prime}(x)\right|^{s} \\
& \geq\left(\lambda^{-1}\right)\left[\left(\min _{1 \leq i \leq n} \inf _{z}\left|f_{i}^{\prime}(z)\right|\right) \lambda^{-1}\right]^{s} \delta^{k_{2} s} .
\end{aligned}
$$

Therefore, for any $i_{1} i_{2} \cdots i_{p} \in \Pi_{k_{1}}$ fixed,

$$
\operatorname{card}\left\{i_{1} \cdots i_{p} \cdots i_{q} \in \Pi_{k_{2}}\right\} \leq\left[\lambda^{2+s}\left(\min _{1 \leq i \leq n} \inf _{z}\left|f_{i}^{\prime}(z)\right|\right)^{-s}\right] \cdot\left(\delta^{-s}\right)^{k_{2}-k_{1}} .
$$


Let

$$
C_{2}=\max \left[\lambda, C_{1}^{-1}\left(\min _{1 \leq i \leq n} \inf _{z}\left|f_{i}^{\prime}(z)\right|\right)^{-1} \lambda, \lambda^{2+s}\left(\min _{1 \leq i \leq n} \inf _{z}\left|f_{i}^{\prime}(z)\right|\right)^{-s}\right] .
$$

Then by (2.10)-(2.12), we have shown that $E$ has $s$-structure with constants $\left(C_{2}, \delta, s\right)$.

(3) Directed-graph sets on a transitive graph satisfying SSC:

Let $\left\{K_{i}\right\}_{i=1}^{n}$ be the graph-directed sets on a transitive graph $G=(V, \mathcal{E})$, where $V=\{1, \cdots, n\}$. Let $\mathcal{E}_{i, j}$ denote the set of edges from vertex $i$ to vertex $j$, and $\mathcal{E}_{i, j}^{k}$ the set of sequences of $k$ edges $\left(e_{1}, \ldots, e_{k}\right)$ which form a directed path from vertex $i$ to vertex $j$. Let $\mathcal{E}_{i}=\cup_{k} \cup_{j} \mathcal{E}_{i, j}^{k}$, all the paths starting at vertex $i$.

By the symmetry, we only need to prove that $K_{1}$ has $s$-structure.

Write $r_{e}$ the contracting ratio of $S_{e}$ for $e \in \mathcal{E}$. Let $r_{*}=\min _{e \in \mathcal{E}} r_{e}$ and

$$
D_{*}=\min _{1 \leq i \leq n} \min \left\{d\left(S_{e}\left(K_{j}\right), S_{e^{\prime}}\left(K_{j^{\prime}}\right)\right): e \neq e^{\prime} \text { with } e \in \mathcal{E}_{i, j} \text { and } e^{\prime} \in \mathcal{E}_{i, j^{\prime}}\right\} .
$$

Take $\delta$ small enough such that $\delta<r_{*}$.

For any infinite admissible path $\left(e_{1}, e_{2}, \cdots, e_{m}, \cdots\right)$ starting at vertex 1 , and any $k \geq 1$, we can take the least integer $l(k)$ satisfying $r_{e_{1}} \cdots r_{e_{l(k)}}<\delta^{k}$. In the same way as above, we have

$$
r_{*} \delta^{k} \leq r_{e_{1}} \cdots r_{e_{l(k)}}<\delta^{k}
$$

Let $\Pi^{k}$ be the collection of all the paths $\left(e_{1}, \cdots, e_{l(k)}\right)\left(\in \mathcal{E}_{1}\right)$ defined above.

Then we have

$$
K_{1}=\bigcup_{\left(e_{1}, \cdots, e_{p}\right) \in \Pi^{k}} E_{e_{1} \cdots e_{p}},
$$

where $E_{e_{1} \cdots e_{p}}=S_{e_{1}} \circ \cdots \circ S_{e_{p}}\left(K_{j}\right)$ with edge $e_{p}$ ending at some vertex $j$.

By the strong separation condition, we have $D_{*}>0$ and for $\left(e_{1}, \cdots, e_{p}\right) \in \Pi^{k}$,

$$
d\left(E_{e_{1} \cdots e_{p}}, K_{1} \backslash E_{e_{1} \cdots e_{p}}\right) \geq D_{*} r_{*} \delta^{k} \text { and }\left|E_{e_{1} \cdots e_{p}}\right| \leq \delta^{k} \cdot \max _{1 \leq i \leq n}\left|K_{i}\right| .
$$

Fix a path $\left(e_{1}, \cdots, e_{p}\right) \in \Pi^{k_{1}}$ and $k_{1}<k_{2}$, we estimate the cardinality of the set

$$
\left\{\left(e_{1}, \cdots, e_{p}, \cdots, e_{q}\right) \in \Pi^{k_{2}}\right\} .
$$

In fact, let $s$ be the Hausdorff dimension of $K_{i}$, we have

$$
\mathcal{H}^{s}\left(E_{e_{1} \cdots e_{p}}\right)=\sum_{\left(e_{1}, \cdots, e_{p}, \cdots, e_{q}\right) \in \Pi^{k_{2}}} \mathcal{H}^{s}\left(E_{e_{1} \ldots e_{p} \ldots e_{q}}\right)
$$

where

$$
\begin{aligned}
\mathcal{H}^{s}\left(E_{e_{1} \cdots e_{p}}\right) & \leq \delta^{k_{1} s} \cdot \max _{1 \leq i \leq n} \mathcal{H}^{s}\left(K_{i}\right), \\
\mathcal{H}^{s}\left(E_{e_{1} \cdots e_{p} \cdots e_{q}}\right) & \geq r_{*}^{s} \delta^{k_{2} s} \cdot \min _{1 \leq i \leq n} \mathcal{H}^{s}\left(K_{i}\right) .
\end{aligned}
$$

Therefore, for $\left(e_{1}, \cdots, e_{p}\right) \in \Pi^{k_{1}}$ fixed,

$\operatorname{card}\left\{\left(e_{1}, \ldots, e_{p}, \ldots, e_{q}\right) \in \Pi^{k_{2}}\right\} \leq\left(\delta^{-s}\right)^{k_{2}-k_{1}}\left(r_{*}^{-s} \max _{1 \leq i \leq n} \mathcal{H}^{s}\left(K_{i}\right) / \min _{1 \leq i \leq n} \mathcal{H}^{s}\left(K_{i}\right)\right)$.

Let

$$
C_{3}=\max \left(D_{*}^{-1} r_{*}^{-1}, \max _{1 \leq i \leq n}\left|K_{i}\right|, r_{*}^{-s} \max _{1 \leq i \leq n} \mathcal{H}^{s}\left(K_{i}\right) / \min _{1 \leq i \leq n} \mathcal{H}^{s}\left(K_{i}\right)\right)
$$

then $K_{1}$ has the $s$-structure with constants $\left(C_{3}, \delta, s\right)$.

(4) Homogeneous Moran set in $\mathcal{M}(J, \bar{n}, \bar{c})$ 
Suppose $E \in \mathcal{M}(J, \bar{n}, \bar{c})$ with dimension $s=-\log \bar{n} / \log \bar{c}$. Since $\bar{n} \bar{c}<1$, we can take $\varepsilon>0$ small enough such that

$$
\bar{n} \bar{c}+(\bar{n}+1) \varepsilon<1 .
$$

Given $k \geq 1, \cup_{i=1}^{t} I_{i}$ is called a $\varepsilon$-block of order $k$, if

(1) $I_{1}, I_{2}, \cdots, I_{t}$ are basic elements of order $k$, having length $(\bar{c})^{k}$;

(2) $I_{1}, I_{2}, \cdots, I_{t}$ are intervals arranged from left to right such that

$$
d\left(I_{p}, I_{p+1}\right) \leq \varepsilon(\bar{c})^{k} \text { for every } p ;
$$

(3) $d\left(\cup_{i=1}^{t} I_{i}, E \backslash \cup_{i=1}^{t} I_{i}\right)>\varepsilon(\bar{c})^{k}$.

Since $\bar{n} \bar{c}+(\bar{n}+1) \varepsilon<1$, for any $\varepsilon$-block $\cup_{i=1}^{t} I_{i}$, we have

$$
t \leq 2 \bar{n} \text {. }
$$

For each $\varepsilon$-block $\cup_{i=1}^{t} I_{i}$ of order $k_{1}$, the number of $\varepsilon$-block of order $k_{2}$ contained in $\cup_{i=1}^{t} I_{i}$ is less than or equal to

$$
t \cdot(\bar{n})^{k_{2}-k_{1}} \leq(2 \bar{n})\left[(\bar{c})^{-s}\right]^{k_{2}-k_{1}} .
$$

Notice that the diameter

$$
\left|\cup_{i=1}^{t} I_{i}\right| \leq[t+(t-1) \varepsilon](\bar{c})^{k} \leq(2 t) \cdot(\bar{c})^{k} \leq(4 \bar{n})(\bar{c})^{k} .
$$

On the other hand, for distinct $\varepsilon$-blocks of order $k$, their distance is greater than $\varepsilon(\bar{c})^{k}$. Then the decomposition with respect to $\varepsilon$-blocks implies that this Moran set has $s$-structure with constants $\left(\max \left(4 \bar{n}, \varepsilon^{-1}\right), \bar{c}, s\right)$, where $s=-\log \bar{n} / \log \bar{c}$.

\section{Proof of Proposition 2}

Let $s(C, \delta)$ be the collection of all the sets having $s$-structure with constants $(C, \delta, s)$ as in Definition 3.

Lemma 2. Suppose $E \in s(C, \delta)$. Then $E \in s\left(C, \delta^{n}\right)$ for any $n \geq 1$.

Proof. For any $k, n \geq 1$, given a word $i_{1} \cdots i_{k n}$, of length $k n$, construct a new word by the following way

$$
\left(i_{1} \cdots i_{n}\right)\left(i_{n+1} \cdots i_{2 n}\right) \cdots\left(i_{(k-1) n+1} \cdots i_{k n}\right),
$$

where each segment $\left(i_{j n+1} \cdots i_{(j+1) n}\right)(0 \leq j \leq k-1)$ is regarded as a new letter. Then this new word has length $k$. It follows from the definition that $E \in s\left(C, \delta^{n}\right)$ for any $n \geq 1$.

Now we are turn to the proof of Proposition 2. Without loss of generality, we assume $|F| \geq 1$. Suppose $E \in s(C, \delta)$. Fixing $C$, by Lemma 2, we can choose $\delta$ small enough such that

$$
C \delta^{t-s}<\left(20^{t} C_{F}\right)^{-1}
$$

where $C_{F}$ is the constant such that for any $y \in F$ and $r<|F|$,

$$
r^{t} \leq \mu(B(y, r)) \leq C_{F} r^{t}
$$

Without loss of generality, for each $k \geq 0$ and each $i_{1} \cdots i_{k} \in \Phi_{k}$, let $n_{i_{1} \cdots i_{k}}\left(\leq C \delta^{-s}\right)$ be the integer such that

$$
\left[1, n_{i_{1} \cdots i_{k}}\right] \cap \mathbb{N}=\left\{j: i_{1} \cdots i_{k} j \in \Phi_{k+1}\right\} .
$$


Fix $y^{*} \in F$. As in Lemma 2.1 of [12], using 5r-covering theorem [11], we can find $m_{0}$ disjoint balls $B\left(y_{i}, 2 \delta\right) \subset B\left(y^{*}, 1 / 2\right)=B\left(y^{*}, \delta^{0} / 2\right)$ with $y_{i} \in F(i=1,2, \ldots, m)$ such that

$$
B\left(y^{*}, 1 / 2\right) \subset \cup_{i=1}^{m_{0}} B\left(y_{i}, 10 \delta\right) .
$$

Therefore,

$$
m_{0} \cdot\left(10^{t} C_{F}\right) \delta^{t} \geq \sum_{i=1}^{m} \mu\left[B\left(y_{i}, 10 \delta\right)\right] \geq \mu\left[B\left(y^{*}, 1 / 2\right)\right] \geq(1 / 2)^{t},
$$

which yields

$$
m_{0} \geq\left(20^{t} C_{F}\right)^{-1} \delta^{-t}>C \delta^{-s} .
$$

Since $\Phi_{1}=\left\{1, \cdots, n_{\emptyset}\right\}$ with $n_{\emptyset}=\operatorname{card}\left(\Phi_{1}\right) \leq C \delta^{-s}$, from the family of balls $\left\{B\left(y_{i}, \delta\right)\right\}_{i=1}^{m_{0}}$, we may select balls $\left\{B\left(y_{i_{1}}, \delta\right)\right\}_{i_{1} \in \Phi_{1}}$ with $y_{i_{1}} \in F$.

By induction on $k$, assume that we get a ball $B\left(y_{i_{1} \cdots i_{k}}, \delta^{k}\right)$ with $i_{1} \cdots i_{k} \in \Phi_{k}$ and $y_{i_{1} \cdots i_{k}} \in F$. Using 5 r-covering theorem again, we have $m_{i_{1} \cdots i_{k}}$ disjoint balls $B\left(y_{i_{1} \cdots i_{k} j}, 2 \delta^{k+1}\right) \subset B\left(y_{i_{1} \cdots i_{k}}, \delta^{k} / 2\right)$ with $y_{i_{1} \cdots i_{k} j} \in F\left(j=1,2, \ldots, m_{i_{1} \cdots i_{k}}\right)$ such that

$$
B\left(y_{i_{1} \cdots i_{k}}, \delta^{k} / 2\right) \subset \cup_{j=1}^{m_{i_{1} \cdots i_{k}}} B\left(y_{i_{1} \cdots i_{k} j}, 10 \delta^{k+1}\right) .
$$

As in the discussion above, we have

$$
m_{i_{1} \cdots i_{k}} \geq\left(20^{t} C_{F}\right)^{-1} \delta^{-t}>C \delta^{-s} \geq n_{i_{1} \cdots i_{k}} .
$$

Thus we can choose a family $\left\{B\left(y_{i_{1} \cdots i_{k} j}, \delta^{k+1}\right)\right\}_{i_{1} \cdots i_{k} j \in \Phi_{k+1}}$ of balls such that

$$
B\left(y_{i_{1} \cdots i_{k} j}, \delta^{k+1}\right) \subset B\left(y_{i_{1} \cdots, i_{k}}, \delta^{k}\right),
$$

and for any $i_{k+1} \neq i_{k+1}^{\prime}$ with $i_{1} \cdots i_{k} i_{k+1}, i_{1} \cdots i_{k} i_{k+1}^{\prime} \in \Phi_{k+1}$,

$$
d\left(B\left(y_{i_{1} i_{2} \ldots i_{k} i_{k+1}}, \delta^{k+1}\right), B\left(y_{i_{1} i_{2} \ldots i_{k} i_{k+1}^{\prime}}, \delta^{k+1}\right)\right) \geq \delta^{k+1} .
$$

Setting

$$
F^{\prime}=\bigcap_{k=1}^{\infty} \bigcup_{i_{1} i_{2} \ldots i_{k} \in \Phi_{k}}\left[B\left(y_{i_{1} i_{2} \ldots i_{k}}, \delta^{k}\right) \cap F\right]
$$

then $F^{\prime} \subset F$.

For any $x \in E$, there is a unique infinite sequence $i_{1}(x) \cdots i_{k}(x) \cdots$ satisfying $i_{1}(x) \cdots i_{k}(x) \in \Phi_{k}$ for each $k$, and

$$
\{x\}=\cap_{k \geq 1} E^{i_{1}(x) \cdots i_{k}(x)} .
$$

Then a bijection $f$ from $E$ to $F^{\prime}$ is defined by

$$
\{f(x)\}=\cap_{k \geq 1}\left[B\left(y_{i_{1}(x) \cdots i_{k}(x)}, \delta^{k}\right) \cap F\right],
$$

where $B\left(y_{i_{1} \cdots i_{k} i_{k+1}}, \delta^{k+1}\right) \subset B\left(y_{i_{1} \cdots i_{k}}, \delta^{k}\right)$ for every $k$.

It suffices to show $f$ is a bilipschitz mapping. Suppose $x, x^{\prime}$ are distinct points of $E$ and

$$
x \in E^{i_{1} \cdots i_{k} i_{k+1}} \text { and } x^{\prime} \in E^{i_{1} \cdots i_{k} i_{k+1}^{\prime}} \text { with } i_{k+1} \neq i_{k+1}^{\prime} \text {. }
$$

Then

$$
C^{-1} \delta^{k+1} \leq d\left(E^{i_{1} \cdots i_{k} i_{k+1}}, E^{i_{1} \cdots i_{k} i_{k+1}^{\prime}}\right) \leq\left|x-x^{\prime}\right| \leq\left|E^{i_{1} \cdots i_{k}}\right| \leq C \delta^{k} .
$$


On the other hand,

$$
\begin{aligned}
\delta^{k+1} & \leq d\left(B\left(y_{i_{1} \cdots i_{k} i_{k+1}}, \delta^{k+1}\right), B\left(y_{i_{1} \cdots i_{k} i_{k+1}^{\prime}}, \delta^{k+1}\right)\right) \\
& \leq\left|f(x)-f\left(x^{\prime}\right)\right| \\
& \leq\left|B\left(y_{i_{1} \cdots i_{k}}, \delta^{k}\right)\right| \leq 2 \delta^{k},
\end{aligned}
$$

which concludes

$$
C^{-1} \delta \leq\left|f(x)-f\left(x^{\prime}\right)\right| /\left|x-x^{\prime}\right| \leq 2 C \delta^{-1}
$$

\section{Proofs of Theorem 1 And Proposition 3}

To prove Theorem 1, it suffices to prove Proposition 3. In fact, let $t=\operatorname{dim}_{H} E_{2}>$ $\operatorname{dim}_{H} E_{1}$, since $E_{1}$ satisfies SSC, applying Proposition 2 and Proposition 1.(2), we only need to show that for any $\varepsilon>0, E_{2}$ includes a $t^{\prime}$-regular subset with $t^{\prime} \in(t-\varepsilon, t]$.

\section{Proof of Proposition 3:}

Since $F$ is a self-similar set, we have

$$
\operatorname{dim}_{H} F=\operatorname{dim}_{B} F=t,
$$

where $\operatorname{dim}_{B}(\cdot)$ is the Box dimension.

Without loss of generality, we assume $|F|=1$ and $F \subset \mathbb{R}^{n}$.

Let $\mathcal{Q}_{k}$ be the collection of all 2 -adic cubes with sidelength $2^{-k}$, i.e.,

$$
\mathcal{Q}_{k}=\left\{Q: Q=\prod_{i=1}^{n}\left[\frac{a_{i}}{2^{k}}, \frac{a_{i}+1}{2^{k}}\right] \text { with } a_{i} \in \mathbb{Z} \text { for all } i\right\} .
$$

Let

$$
N_{k}=\operatorname{card}\left\{Q \in \mathcal{Q}_{k}: F \cap Q \neq \varnothing\right\} .
$$

By the definition of Box dimension, we have

$$
\lim _{k \rightarrow \infty} \frac{\log N_{k}}{\log 2^{k}}=t
$$

For any fixed $k$, we get a collection of cubes with sidelength $2^{-k}$ which intersect $F$. Therefore there is a constant $C(n)$ only depending on $n$ such that we can select a subset $\Upsilon_{k}$ of $\left\{Q \in \mathcal{Q}_{k}: F \cap Q \neq \varnothing\right\}$ such that

$$
\operatorname{card}\left(\Upsilon_{k}\right) \geq N_{k} / C(n)
$$

and

$$
Q_{1} \cap Q_{2}=\varnothing
$$

for any distinct elements $Q_{1}, Q_{2}$ in $\Upsilon_{k}$. Then (4.1) implies

$$
d\left(Q_{1}, Q_{2}\right) \geq 2^{-k} \text {. }
$$

For any $Q \in \Upsilon_{k}$, take a point $x_{Q} \in F \cap Q$ and an infinite sequence $j_{1} \cdots j_{t} \cdots$ satisfying

$$
\left\{x_{Q}\right\}=\cap_{t \geq 1} S_{j_{1} \cdots j_{t}}(F) .
$$

Find an index $t_{Q}$ such that the ratio of $S_{j_{1} \cdots j_{t_{Q}}}$ belongs to

$$
\left[r \cdot 2^{-k} / 3,2^{-k} / 3\right]
$$

where $r$ is the least ratio of similitudes with respect to $F$. Write $\mathbf{S}_{\mathbf{j}_{Q}}=S_{j_{1} \cdots j_{t_{Q}}}$, and let $r_{Q}$ denote the ratio of $\mathbf{S}_{\mathbf{j}_{Q}}$, then $r_{Q} \in\left[r \cdot 2^{-k} / 3,2^{-k} / 3\right]$. 
We shall verify $\left\{S_{\mathbf{j}_{Q}}\right\}_{Q \in \Upsilon_{k}}$ can generate a self-similar set $F_{k}$ satisfying SSC.

In fact, the corresponding invariant set $F_{k}$ is contained in $F$, since

$$
S_{\mathbf{j}_{Q}}(F) \subset F \text { for any } Q \in \Upsilon_{k} .
$$

That means the diameter $\left|F_{k}\right| \leq 1$, and thus

$$
\left|S_{\mathbf{j}_{Q}}\left(F_{k}\right)\right| \leq r_{Q}\left|F_{k}\right| \leq 2^{-k} / 3 .
$$

Therefore, for any distinct elements $Q_{1}, Q_{2}$ in $\Upsilon_{k}$,

$$
\begin{aligned}
d\left(S_{\mathbf{j}_{Q_{1}}}\left(F_{k}\right), S_{\mathbf{j}_{Q_{1}}}\left(F_{k}\right)\right) & \geq d\left(Q_{1}, Q_{2}\right)-\left|S_{\mathbf{j}_{Q_{1}}}\left(F_{k}\right)\right|-\left|S_{\mathbf{j}_{Q_{2}}}\left(F_{k}\right)\right| \\
& \geq 2^{-k}-2\left(2^{-k} / 3\right)=2^{-k} / 3 .
\end{aligned}
$$

Then $F_{k}=\cup_{Q \in \Upsilon_{k}} S_{\mathbf{j}_{Q}}\left(F_{k}\right)$ satisfies SSC, which implies that the Hausdorff dimension $t_{k}$ of $F_{k}$ is determined by

$$
\sum_{Q \in \Upsilon_{k}}\left(r_{Q}\right)^{t_{k}}=1
$$

where $r_{Q} \in\left[r \cdot 2^{-k} / 3,2^{-k} / 3\right]$.

Therefore,

$$
\left[N_{k} / C(n)\right]\left(r 2^{-k} / 3\right)^{t_{k}} \leq \operatorname{card}\left(\Upsilon_{k}\right)\left(r 2^{-k} / 3\right)^{t_{k}} \leq \sum_{Q \in \Upsilon_{k}}\left(r_{Q}\right)^{t_{k}}=1 .
$$

As a result,

$$
t_{k} \geq \frac{\log \left[N_{k} / C(n)\right]}{\log \left[2^{k}(3 / r)\right]} .
$$

Take $k$ large enough, we have

$$
t_{k}>t-\varepsilon .
$$

Now, we get a self-similar set $F_{k}$ satisfying SSC such that $F_{k} \subset F$. Here $F_{k}$ is $t_{k}$-regular with $t_{k} \in(t-\varepsilon, t]$.

The proof of Proposition 3 is finished.

\section{Proof of Theorem 3}

\subsection{Bilipschitz Equivalence of Self-similar Sets with SSC.}

Let $E, F$ be self-similar sets satisfying SSC, with ratios sets $\left\{r_{i}\right\}_{i=1}^{n}$ and $\left\{t_{j}\right\}_{j=1}^{m}$ respectively, satisfying

Write $\rho_{j}=t_{j}^{s}$ for $j=1, \cdots, m$.

$$
\sum_{i=1}^{n} r_{i}^{s}=\sum_{j=1}^{m} t_{j}^{s}=1 .
$$

Recall that $\Sigma_{m}^{*}$ is the set of all finite words composed of $1, \cdots, m$. Let $\Sigma_{m}=$ $\{1, \cdots, m\}^{\infty}$ be a symbolic system equipped with the Bernoulli measure $v=$ $\left(\rho_{1}, \cdots, \rho_{m}\right)$. The cylinder $\left[i_{1} \cdots i_{l}\right]$ generated by the word $i_{1} \cdots i_{l}$ is defined by $\left[i_{1} \cdots i_{l}\right]=\left\{j_{1} \cdots j_{l} \cdots \in \Sigma_{m}: j_{1} \cdots j_{l}=i_{1} \cdots i_{l}\right\}$. Then for each cylinder $\left[i_{1} \cdots i_{l}\right]$,

$$
v\left(\left[i_{1} \cdots i_{l}\right]\right)=\Pi_{t=1}^{l} \rho_{i_{t}} .
$$

Given a word $i_{1} \cdots i_{k}$ and a subset $B$ of $\Sigma_{m}$, let

$$
A=i_{1} \cdots i_{k} B=\left\{i_{1} \cdots i_{k} j_{1} \cdots j_{l} \cdots: j_{1} \cdots j_{l} \cdots \in B\right\}
$$

and denote by $A \prec B$. 
Lemma 3. [21] Suppose E, F are self-similar sets satisfying SSC, with ratios sets $\left\{r_{i}\right\}_{i=1}^{n}$ and $\left\{t_{j}\right\}_{j=1}^{m}$ respectively, where $\sum_{i=1}^{n} r_{i}^{s}=\sum_{j=1}^{m} t_{j}^{s}=1$. Let $v$ be the Bernoulli measure $\left(t_{1}^{s}, \cdots, t_{m}^{s}\right)$ on $\Sigma_{m}$. Then $E$ and $F$ are bilipschitz equivalent if and only if there are $\Omega_{1}, \Omega_{2}, \cdots, \Omega_{k},\left\{\Delta_{i, j}\right\}_{1 \leq i \leq k, 1 \leq j \leq n}$, which are unions of finitely many cylinders in $\Sigma_{m}$, satisfying

$$
\begin{array}{cccccc}
\Omega_{1} & = & \Delta_{1,1} & \cup \Delta_{2,1} & \cdots & \cup \Delta_{1, n} \\
\Omega_{2} & = & \Delta_{2,1} & \cup \Delta_{2,2} & \cdots & \cup \Delta_{2, n} \\
\vdots & \vdots & \vdots & \vdots & \ddots & \vdots \\
\Omega_{k} & = & \Delta_{k, 1} & \cup \Delta_{k, 2} & \cdots & \cup \Delta_{k, n}
\end{array}
$$

where

(1) right side of every equality above is a disjoint union;

(2) for every $(i, j)$, there exist $\beta \in \Sigma_{m}^{*}$ and $\gamma \in \mathbb{N} \cap[1, k]$ such that $\Delta_{i, j}=\beta \Omega_{\gamma}$;

(3) for every $(i, j)$,

$$
\frac{v\left(\Delta_{i, j}\right)}{v\left(\Omega_{i}\right)}=r_{j}^{s} .
$$

\subsection{Proof of Theorem 3.}

We assume that $F, F^{\prime}$ are self-similar sets satisfying SSC and $\operatorname{dim}_{H} F=\operatorname{dim}_{H} F^{\prime}=$ $s$. Let $\left\{r_{i}\right\}_{i=1}^{n},\left\{t_{j}\right\}_{j=1}^{m}$ be the corresponding ratio sets for $F$ and $F^{\prime}$ respectively.

Let $K \subset F, K^{\prime} \subset F^{\prime}$ with $\mathcal{H}^{s}(K), \mathcal{H}^{s}\left(K^{\prime}\right)>0$ such that $K$ and $K^{\prime}$ are bilipschitz equivalent with the corresponding bilipschitz bijection $f: K \rightarrow K^{\prime}$ satisfying

$$
C^{-1}|x-y| \leq|f(x)-f(y)| \leq C|x-y| \text { for all } x, y \in K .
$$

We will show that $F$ and $F^{\prime}$ are bilipschitz equivalent.

Suppose $H=\cup_{i=1}^{n} S_{i}(H)$ is a self-similar set satisfying SSC. For $j^{*}=j_{1} \cdots j_{k} \in$ $\Sigma_{n}^{*}$, write $S_{j^{*}}=S_{j_{1}} \circ \cdots \circ S_{j_{k}}$ and $H_{j^{*}}=S_{j^{*}}(H)$. $H_{j^{*}}$ is called a fine copy of $H$.

Lemma 4. Suppose $F^{\prime}$ is generated by $m$ similitudes with ratios $\left\{t_{j}\right\}_{j=1}^{m}$. Then there is an integer $N$ such that for any fine copy $\bar{F}$ of $F$, there exists a subset $\Lambda \subset\{1, \cdots, m\}^{N}$ so that

$$
f(\bar{F} \cap K)=\bigcup_{j^{*} \in \Lambda}\left(F_{j_{1} \cdots j_{k} j^{*}}^{\prime} \cap K^{\prime}\right),
$$

where $F_{j_{1} \cdots j_{k}}^{\prime}$ is the smallest fine copy containing $f(\bar{F} \cap K)$.

Proof. Take $N$ large enough such that

$$
\left(\max _{j} t_{j}\right)^{N}<\left[\min _{i_{1} \neq i_{2}} d\left(F_{i_{1}}, F_{i_{2}}\right) /|F|\right] \cdot\left[\min _{l_{1} \neq l_{2}} d\left(F_{l_{1}}^{\prime}, F_{l_{2}}^{\prime}\right) /\left|F^{\prime}\right|\right] / C^{2} .
$$

We will show $N$ is the integer desired.

Suppose on the contrary, there exist $y_{1}, y_{2}$ and a fine copy $F_{j_{1} \cdots j_{k} j^{*}}^{\prime}\left(\left|j^{*}\right|=N\right)$ containing $y_{1}, y_{2}$ such that $y_{1} \in f(\bar{F} \cap K), y_{2} \in K^{\prime} \backslash f(\bar{F} \cap K)$. Thus

$$
\begin{aligned}
\left(\max _{j} t_{j}\right)^{N}\left|F_{j_{1} \cdots j_{k}}^{\prime}\right| & \geq\left|F_{j_{1} \cdots j_{k} j^{*}}^{\prime}\right| \geq\left|y_{1}-y_{2}\right| \\
& \geq C^{-1} d(\bar{F} \cap K, K \backslash \bar{F}) \geq C^{-1} d(\bar{F}, F \backslash \bar{F}) \\
& \geq C^{-1}\left[\min _{i_{1} \neq i_{2}} d\left(F_{i_{1}}, F_{i_{2}}\right) /|F|\right]|\bar{F}| .
\end{aligned}
$$


Since $F_{j_{1} \ldots j_{k}}^{\prime}$ is the smallest fine copy containing $f(\bar{F} \cap K)$, there are distinct $j$ and $j^{\prime}$ such that $f(\bar{F} \cap K) \cap F_{j_{1} \cdots j_{k} j}^{\prime} \neq \varnothing, f(\bar{F} \cap K) \cap F_{j_{1} \cdots j_{k} j^{\prime}}^{\prime} \neq \varnothing$. Therefore,

$$
\begin{aligned}
|\bar{F}| \geq|\bar{F} \cap K| & \geq C^{-1}|f(\bar{F} \cap K)| \\
& \geq C^{-1} \min _{j_{k+1} \neq j_{k+1}^{\prime}} d\left(F_{j_{1} \cdots j_{k} j_{k+1}}^{\prime}, F_{j_{1} \cdots j_{k} j_{k+1}^{\prime}}^{\prime}\right) \\
& \geq C^{-1}\left(\left|F_{j_{1} \cdots j_{k}}^{\prime}\right| /\left|F^{\prime}\right|\right) \min _{l_{1} \neq l_{2}} d\left(F_{l_{1}}^{\prime}, F_{l_{2}}^{\prime}\right) .
\end{aligned}
$$

Therefore,

$$
\left(\max _{j} t_{j}\right)^{N}\left|F_{j_{1} \cdots j_{k}}^{\prime}\right| \geq\left[\min _{i_{1} \neq i_{2}} d\left(F_{i_{1}}, F_{i_{2}}\right) /|F|\right]\left[\min _{l_{1} \neq l_{2}} d\left(F_{l_{1}}^{\prime}, F_{l_{2}}^{\prime}\right) /\left|F^{\prime}\right|\right]\left|F_{j_{1} \cdots j_{k}}^{\prime}\right| / C^{2}
$$

which implies

$$
\left(\max _{j} t_{j}\right)^{N} \geq\left[\min _{i_{1} \neq i_{2}} d\left(F_{i_{1}}, F_{i_{2}}\right) /|F|\right]\left[\min _{l_{1} \neq l_{2}} d\left(F_{l_{1}}^{\prime}, F_{l_{2}}^{\prime}\right) /\left|F^{\prime}\right|\right] / C^{2},
$$

which contradicts (5.1).

Let $N$ be the integer defined in Lemma 4 .

For a fine copy $F_{\mathbf{i}}$ with $F_{\mathbf{i}} \cap K \neq \varnothing$, we get a decomposition

$$
F_{\mathbf{i}}=\cup_{u=1}^{n} F_{\mathbf{i} * u} .
$$

Then by Lemma 4 , there is a subset $\Lambda_{\mathbf{i}} \subset\{1, \cdots, m\}^{N}$ such that

$$
f\left(F_{\mathbf{i}} \cap K\right)=\bigcup_{j^{*} \in \Lambda_{\mathbf{i}}}\left(F_{\mathbf{j}(\mathbf{i}) * j^{*}}^{\prime} \cap K^{\prime}\right)
$$

where $F_{\mathbf{j}(\mathbf{i})}^{\prime}$ is the smallest fine copy containing $f\left(F_{\mathbf{i}} \cap K\right)$. Let

$$
\bar{\Omega}_{\mathbf{i}}=\cup_{j^{*} \in \Lambda_{\mathbf{i}}}\left[j^{*}\right],
$$

where $\left[j^{*}\right]$ is the cylinder.

Notice that



Suppose that $F_{\mathbf{j}(\mathbf{i}) * \bar{\beta}(\mathbf{i}, u)}^{\prime}$ is the smallest fine copy containing $f\left(F_{\mathbf{i} * u} \cap K\right)$ with $\bar{\beta}(\mathbf{i}, u) \in \Sigma_{m}^{*}$. Then by Lemma 4 and (5.2), we have

$$
\cup_{u=1}^{n} f\left(F_{\mathbf{i} * u} \cap K\right)=\cup_{u=1}^{n}\left[\bigcup_{v^{*} \in \Lambda_{\mathbf{i} * u}} F_{\mathbf{j}(\mathbf{i}) * \bar{\beta}(\mathbf{i}, u) * v^{*}}^{\prime} \cap K^{\prime}\right] .
$$

It follows from (5.4) and (5.5) that

$$
\bigcup_{j^{*} \in \Lambda_{\mathbf{i}}}\left(F_{\mathbf{j}(\mathbf{i}) * j^{*}}^{\prime} \cap K^{\prime}\right)=\cup_{u=1}^{n}\left[\bigcup_{v^{*} \in \Lambda_{\mathbf{i} * u}} F_{\mathbf{j}(\mathbf{i}) * \bar{\beta}(\mathbf{i}, u) * v^{*}}^{\prime} \cap K^{\prime}\right] .
$$

Let $\bar{\Delta}_{\mathbf{i}, u}=\cup_{v^{*} \in \Lambda_{\mathbf{i} * u}}\left[\bar{\beta}(\mathbf{i}, u) * v^{*}\right]$, then

$$
\bar{\Delta}_{\mathbf{i}, u}=\bar{\beta}(\mathbf{i}, u)\left(\cup_{v^{*} \in \Lambda_{\mathbf{i} * u}}\left[v^{*}\right]\right)=\bar{\beta}(\mathbf{i}, u) \bar{\Omega}_{\mathbf{i} * u} .
$$

By (5.6), we have

$$
\bigcup_{j^{*} \in \Lambda_{\mathbf{i}}}\left[\mathbf{j}(\mathbf{i}) * j^{*}\right]=\cup_{u=1}^{n} \bigcup_{v^{*} \in \Lambda_{\mathbf{i} * u}}\left[\mathbf{j}(\mathbf{i}) * \bar{\beta}(\mathbf{i}, u) * v^{*}\right],
$$

i.e.,

which implies

$$
\bigcup_{j^{*} \in \Lambda_{\mathbf{i}}}\left[j^{*}\right]=\cup_{u=1}^{n} \bigcup_{v^{*} \in \Lambda_{\mathbf{i} * u}}\left[\bar{\beta}(\mathbf{i}, u) * v^{*}\right],
$$

$$
\bar{\Omega}_{\mathbf{i}}=\cup_{u=1}^{n} \bar{\Delta}_{\mathbf{i}, u}
$$


Notice that (5.8) is a disjoint union, and

$$
\bar{\Delta}_{\mathbf{i}, u} \prec \bar{\Omega}_{\mathbf{i} * u} \text { for all } u \text {. }
$$

Let $\bar{v}$ be Borel measure defined by

$$
\bar{v}(A)=\mathcal{H}^{s}(f(A)) \text { for all } A \subset K .
$$

Since $f: K \rightarrow K^{\prime}$ is bilipschitz, $\bar{v}$ is an absolutely continuous measure with respect to $\left.\mathcal{H}^{s}\right|_{K}$. Therefore, there exists Radon-Nikodym derivative

$$
D f(x)=\lim _{r \rightarrow 0} \frac{\mathcal{H}^{s}(f(K \cap B(x, r))}{\mathcal{H}^{s}(K \cap B(x, r))} \text { for } \mathcal{H}^{s} \text {-almost all } x \in K,
$$

such that for any Borel set $A \subset K$,

$$
\mathcal{H}^{s}(f(A))=\left.\int_{A} D f(x) d \mathcal{H}^{s}\right|_{K}(x) .
$$

Let $a>0$ be the essential supremum of $D f$, i.e.,

$$
a:=\inf \left\{b: \mathcal{H}^{s}\{x \in K: D f(x) \geq b\}=0\right\} .
$$

Then for any Borel set $A \subset K$,

$$
\mathcal{H}^{s}(f(A)) \leq a \mathcal{H}^{s}(A)
$$

Recall that $C$ is the bilipschitz constant of $f$, by the property of the Hausdorff measure, $\mathcal{H}^{s}\left(f(K \cap B(x, r)) \leq C^{s} \cdot \mathcal{H}^{s}(K \cap B(x, r))\right.$, so $D f(x) \leq C^{s}$ for $\mathcal{H}^{s}$-almost all $x \in K$. It follows that $a \leq C^{s}<\infty$.

Lemma 5. Let $c>0$ and suppose $D$ is a fine copy of $F$, satisfying

$$
D \subset B(x, r) \text { and }|D| \geq c \cdot r,
$$

where the ball $B(x, r)$ satisfies

$$
\left(1-\frac{\mathcal{H}^{s}(B(x, r) \cap K)}{\mathcal{H}^{s}(B(x, r) \cap F)}\right) \leq \varsigma \text { and }\left(a-\frac{\mathcal{H}^{s}(f(B(x, r) \cap K))}{\mathcal{H}^{s}(B(x, r) \cap K)}\right) \leq \varepsilon .
$$

Then there is a constant $c^{*}>0$ only depending on $c$ and $F$ such that

$$
\begin{gathered}
\left(1-\frac{\mathcal{H}^{s}(D \cap K)}{\mathcal{H}^{s}(D)}\right) \leq c^{*} \varsigma, \\
\left(a-\frac{\mathcal{H}^{s}(f(D \cap K))}{\mathcal{H}^{s}(D \cap K)}\right) \leq c^{*}\left[1-c^{*} \varsigma\right]^{-1} \varepsilon .
\end{gathered}
$$

Proof. The self-similar set $F$ with SSC, equipped with Hausdorff measure $\left.\mathcal{H}^{s}\right|_{F}$, is $s$-regular. That means for any $x \in F$ and $r \leq|F|$,

$$
\mathcal{H}^{s}(B(x, r) \cap F) \leq C_{F} r^{s}
$$

for some constant $C_{F}>0$. Suppose $D=S(F)$, where $S$ is a similitude with ratio $r_{D}$. Then $\mathcal{H}^{s}(D)=r_{D}^{s} \mathcal{H}^{s}(F),|D|=r_{D}|F|$, which implies

$$
\begin{aligned}
\mathcal{H}^{s}(D) & =\left[|D|^{s} \mathcal{H}^{s}(F)\right] /|F|^{s} \geq\left[c^{s} \mathcal{H}^{s}(F) /|F|^{s}\right] r^{s} \\
& \geq\left[c^{s} \mathcal{H}^{s}(F) /\left(|F|^{s} C_{F}\right)\right] \mathcal{H}^{s}(B(x, r) \cap F) .
\end{aligned}
$$

Let $c^{*}=\left(|F|{ }^{s} C_{F}\right) /\left(c^{s} \mathcal{H}^{s}(F)\right)$, where $c^{*}$ only depends on $c$ and $F$. Then

$$
\mathcal{H}^{s}(D) \geq\left(c^{*}\right)^{-1} \mathcal{H}^{s}(B(x, r) \cap F),
$$


Notice that

$$
\begin{aligned}
& {\left[1-\frac{\mathcal{H}^{s}(D \cap K)}{\mathcal{H}^{s}(D)}\right] \cdot \mathcal{H}^{s}(D) } \\
= & \mathcal{H}^{s}(D \backslash K) \\
\leq & \mathcal{H}^{s}((B(x, r) \cap F) \backslash K) \\
= & \mathcal{H}^{s}(B(x, r) \cap F)-\mathcal{H}^{s}(B(x, r) \cap K) \\
\leq & {\left[1-\frac{\mathcal{H}^{s}(B(x, r) \cap K)}{\mathcal{H}^{s}(B(x, r) \cap F)}\right] \cdot \mathcal{H}^{s}(B(x, r) \cap F) . }
\end{aligned}
$$

Then (5.11) follows from (5.13).

Notice that

$$
\frac{\mathcal{H}^{s}(f(D \cap K))+\mathcal{H}^{s}(f[B(x, r) \cap(K \backslash D)])}{\mathcal{H}^{s}(D \cap K)+\mathcal{H}^{s}(B(x, r) \cap(K \backslash D))}=\frac{\mathcal{H}^{s}(f(B(x, r) \cap K))}{\mathcal{H}^{s}(B(x, r) \cap K)} .
$$

By (5.10), we have

$$
\mathcal{H}^{s}(f[B(x, r) \cap(K \backslash D)]) \leq a \cdot \mathcal{H}^{s}[B(x, r) \cap(K \backslash D)] .
$$

From (5.11), we also have

$$
\begin{aligned}
\mathcal{H}^{s}(D \cap K) & \geq\left[1-c^{*} \varsigma\right] \mathcal{H}^{s}(D) \\
& \geq\left[1-c^{*} \varsigma\right]\left(c^{*}\right)^{-1} \mathcal{H}^{s}(B(x, r) \cap F) \\
& \geq\left[1-c^{*} \varsigma\right]\left(c^{*}\right)^{-1} \mathcal{H}^{s}(B(x, r) \cap K) .
\end{aligned}
$$

It follows from (5.14) and (5.15) that

$$
\begin{aligned}
a-\frac{\mathcal{H}^{s}(f(D \cap K))}{\mathcal{H}^{s}(D \cap K)} & \leq\left(a-\frac{\mathcal{H}^{s}(f(B(x, r) \cap K))}{\mathcal{H}^{s}(B(x, r) \cap K)}\right) \frac{\mathcal{H}^{s}(B(x, r) \cap K)}{\mathcal{H}^{s}(D \cap K)} \\
& \leq c^{*}\left[1-c^{*} \varsigma\right]^{-1} \varepsilon, \quad \text { (using estimation (5.16)) }
\end{aligned}
$$

which yields estimation (5.12).

We can get an estimation similar to (5.11) for fine copy of $F^{\prime}$.

Lemma 6. Let $D^{\prime}$ be a fine copy of $F^{\prime}$, satisfying

$$
D^{\prime} \subset B\left(x^{\prime}, r^{\prime}\right) \text { and }\left|D^{\prime}\right| \geq d \cdot r^{\prime} \text { with } d>0 .
$$

Then there is a constant $d^{*}>0$ only depending on $d$ and $F^{\prime}$ such that

$$
\left(1-\frac{\mathcal{H}^{s}\left(D^{\prime} \cap K^{\prime}\right)}{\mathcal{H}^{s}\left(D^{\prime}\right)}\right) \leq d^{*}\left(1-\frac{\mathcal{H}^{s}\left(B\left(x^{\prime}, r^{\prime}\right) \cap K^{\prime}\right)}{\mathcal{H}^{s}\left(B\left(x^{\prime}, r^{\prime}\right) \cap F^{\prime}\right)}\right) .
$$

As shown above, for a fine copy $F_{\mathbf{i}}$ with $F_{\mathbf{i}} \cap K \neq \varnothing$, we have

$$
\begin{aligned}
f\left(F_{\mathbf{i}} \cap K\right) & =\bigcup_{j^{*} \in \Lambda_{\mathbf{i}}}\left(F_{\mathbf{j}(\mathbf{i}) * j^{*}}^{\prime} \cap K^{\prime}\right), \\
& =\cup_{u=1}^{n}\left[\bigcup_{v^{*} \in \Lambda_{\mathbf{i} * u}} F_{\mathbf{j}(\mathbf{i}) * \bar{\beta}(\mathbf{i}, u) * v^{*}}^{\prime} \cap K^{\prime}\right] .
\end{aligned}
$$

Given $c>0$, let $c^{*}$ be the constant mentioned in Lemma 5. Recall that $C \geq 1$ is the bilipschitz constant of $f: K \rightarrow K^{\prime}$. We denote $\alpha_{t} \asymp \beta_{t}$, if there is a constant $\kappa>0$ independent of $t$ such that for any index $t$,

$$
\kappa \beta_{t} \leq \alpha_{t} \leq \kappa^{-1} \beta_{t}
$$

Keep the notations of (5.18) in the following lemma. 
Lemma 7. Suppose $x \in K, F_{\mathbf{i}} \subset B(x, r)$ with $\left|F_{\mathbf{i}}\right| \geq\left|F_{\mathbf{i} * u}\right| \geq c r$ for every $u$, and

$$
\left(1-\frac{\mathcal{H}^{s}(B(x, r) \cap K)}{\mathcal{H}^{s}(B(x, r) \cap F)}\right) \leq\left(c^{*}\right)^{-1}\left(1-\max _{i} r_{i}^{s}\right) / 2 .
$$

Then we have

$$
\left|F_{\mathbf{i}}\right| \asymp\left|F_{\mathbf{i}} \cap K\right| \asymp\left|F_{\mathbf{j}(\mathbf{i})}^{\prime}\right| \asymp\left|F_{\mathbf{j}(\mathbf{i}) * j^{*}}^{\prime}\right| \asymp\left|F_{\mathbf{j}(\mathbf{i}) * \bar{\beta}(\mathbf{i}, u) * v^{*}}^{\prime}\right| .
$$

As a consequence, there is a constant $\kappa>0$ independent of $c$ such that

$$
\left|F_{\mathbf{j}(\mathbf{i})}^{\prime}\right| \geq\left|F_{\mathbf{j}(\mathbf{i}) * \bar{\beta}(\mathbf{i}, u) * v^{*}}^{\prime}\right| \geq \kappa(c r),
$$

where

$$
F_{\mathbf{j}(\mathbf{i}) * \bar{\beta}(\mathbf{i}, u) * v^{*}}^{\prime} \subset F_{\mathbf{j}(\mathbf{i})}^{\prime} \subset B\left(f(x),\left(C+2 \kappa^{-1}\right) r\right) .
$$

Proof. We conclude that there are at least two distinct letters $j_{1}$ and $j_{2}$ such that

$$
F_{\mathbf{i} * j_{1}} \cap K \neq \varnothing \text { and } F_{\mathbf{i} * j_{2}} \cap K \neq \varnothing .
$$

On the contrary, suppose there exists only one letter $j$ such that $F_{\mathbf{i} * j} \cap K \neq \varnothing$, that means

$$
F_{\mathbf{i}} \cap K \subset F_{\mathbf{i} * j} .
$$

Applying (5.19) to Lemma 5, we have

$$
\begin{aligned}
\left(1-\max _{i} r_{i}^{s}\right) / 2 & \geq 1-\mathcal{H}^{s}\left(F_{\mathbf{i}} \cap K\right) / \mathcal{H}^{s}\left(F_{\mathbf{i}}\right) \\
& =1-\mathcal{H}^{s}\left(F_{\mathbf{i} * j} \cap K\right) / \mathcal{H}^{s}\left(F_{\mathbf{i}}\right) \\
& \geq 1-\mathcal{H}^{s}\left(F_{\mathbf{i} * j}\right) / \mathcal{H}^{s}\left(F_{\mathbf{i}}\right) \\
& \geq 1-\max _{i} r_{i}^{s},
\end{aligned}
$$

this is a contradiction. By (5.23), we have

$$
\left|F_{\mathbf{i}}\right| \geq\left|F_{\mathbf{i}} \cap K\right| \geq d\left(F_{\mathbf{i} * j_{1}}, F_{\mathbf{i} * j_{2}}\right) \geq\left[\left(\min _{i \neq i^{\prime}} d\left(F_{i}, F_{i^{\prime}}\right)\right) /|F|\right] \cdot\left|F_{\mathbf{i}}\right| .
$$

That means

$$
\left|F_{\mathbf{i}}\right| \asymp\left|F_{\mathbf{i}} \cap K\right| \text {. }
$$

On the other hand, since $f$ is bilipschitz, we have

$$
\left|F_{\mathbf{i}} \cap K\right| \asymp\left|f\left(F_{\mathbf{i}} \cap K\right)\right| \text {. }
$$

Notice that $F_{\mathbf{j}(\mathbf{i})}^{\prime}$ is the smallest fine copy of $F^{\prime}$ such that $F_{\mathbf{j}(\mathbf{i})}^{\prime}$ contains $f\left(F_{\mathbf{i}} \cap K\right)$, then there are distinct letters $j_{1}^{\prime}$ and $j_{2}^{\prime}$ such that

$$
F_{\mathbf{j}(\mathbf{i}) * j_{1}^{\prime}}^{\prime} \cap f\left(F_{\mathbf{i}} \cap K\right) \neq \varnothing \text { and } F_{\mathbf{j}(\mathbf{i}) * j_{2}^{\prime}}^{\prime} \cap f\left(F_{\mathbf{i}} \cap K\right) \neq \varnothing
$$

by an analogue discussion to (5.24), we get

$$
\left|f\left(F_{\mathbf{i}} \cap K\right)\right| \asymp\left|F_{\mathbf{j}(\mathbf{i})}^{\prime}\right| .
$$

Since $\left|j^{*}\right|=N$,

$$
\left|F_{\mathbf{j}(\mathbf{i})}^{\prime}\right| \asymp\left|F_{\mathbf{j}(\mathbf{i}) * j^{*}}^{\prime}\right| .
$$

Here $\mathbf{j}(\mathbf{i}) * \bar{\beta}(\mathbf{i}, u)=\mathbf{j}(\mathbf{i} * u)$ and $\left|v^{*}\right|=N$, by similar discussion as above, we have

$$
\left|F_{\mathbf{j}(\mathbf{i}) * \bar{\beta}(\mathbf{i}, u) * v^{*}}^{\prime}\right| \asymp\left|F_{\mathbf{j}(\mathbf{i} * u)}^{\prime}\right| \asymp\left|F_{\mathbf{i} * u}\right| \asymp\left|F_{\mathbf{i}}\right| .
$$

Therefore (5.20) is proved. Suppose $\kappa$ is the constant of (5.20), then

$$
\left|F_{\mathbf{j}(\mathbf{i})}^{\prime}\right|,\left|F_{\mathbf{j}(\mathbf{i}) * \bar{\beta}(\mathbf{i}, u) * v^{*}}^{\prime}\right| \geq \kappa\left|F_{\mathbf{i}}\right| \geq \kappa(c r) .
$$


Given $f(y) \in F_{\mathbf{j}(\mathbf{i})}^{\prime} \cap K^{\prime}$ with $y \in F_{\mathbf{i}} \subset B(x, r)$, we have

$$
|f(y)-f(x)| \leq C|y-x| \leq C r
$$

and thus

$F_{\mathbf{j}(\mathbf{i})}^{\prime} \subset B\left(f(x), C r+\left|F_{\mathbf{j}(\mathbf{i})}^{\prime}\right|\right) \subset B\left(f(x),\left(C r+\kappa^{-1}\left|F_{\mathbf{i}}\right|\right)\right) \subset B\left(f(x),\left(C+2 \kappa^{-1}\right) r\right)$.

Therefore, $F_{\mathbf{j}(\mathbf{i}) * \bar{\beta}(\mathbf{i}, u) * v^{*}}^{\prime} \subset F_{\mathbf{j}(\mathbf{i})}^{\prime} \subset B\left(f(x),\left(C+2 \kappa^{-1}\right) r\right)$.

Remark 6. By (5.20), we have $\left|F_{\mathbf{j}(\mathbf{i})}^{\prime}\right| \asymp\left|F_{\mathbf{j}(\mathbf{i}) * \bar{\beta}(\mathbf{i}, u) * v^{*}}^{\prime}\right|$, which implies that the length of $\bar{\beta}(\mathbf{i}, u)$ is uniformly bounded, i.e., there exists a constant $\vartheta$ independent of $\mathbf{i}, u$ such that

$$
|\bar{\beta}(\mathbf{i}, u)| \leq \vartheta
$$

Given $B(x, r)$ with $x \in K$, we can take a largest fine copy $F_{\mathbf{i}}$ such that

$$
x \in F_{\mathbf{i}} \subset B(x, r) .
$$

Then $\left|F_{\mathbf{i}}\right| \geq c_{1} r$, where constant $c_{1}=\min _{i} r_{i}$. Let

$$
c=c_{1}\left(\min _{i} r_{i}\right)^{2^{\left(m^{N}\right)}}=\left(\min _{i} r_{i}\right)^{2^{\left(m^{N}\right)}+1}
$$

and

$$
d=\left[\kappa /\left(C+2 \kappa^{-1}\right)\right] c
$$

Suppose $c^{*}$ and $d^{*}$ are the corresponding constants as in Lemmas 5 and 6 .

Assume that $\left\{\varepsilon_{k}\right\}_{k},\left\{\varsigma_{k}\right\}_{k}$ and $\left\{r_{k}\right\}_{k}$ are sequences of positive numbers such that

$$
\lim _{k} \varepsilon_{k}=\lim _{k} \varsigma_{k}=\lim _{k} r_{k}=0 .
$$

Here we assume that $\sup _{k} \varsigma_{k}<\left(c^{*}\right)^{-1}\left(1-\max _{i} r_{i}^{s}\right) / 2$.

Let

Then $\lim _{k} \delta_{k}=0$, and

$$
\delta_{k}=\frac{a}{a-c^{*}\left(1-c^{*} \varsigma_{k}\right)^{-1} \varepsilon_{k}}-1 \text {. }
$$

for any $z_{1}, z_{2} \in\left[a-c^{*}\left(1-c^{*} \varsigma_{k}\right)^{-1} \varepsilon_{k}, a\right]$.

$$
\left|z_{1} / z_{2}-1\right| \leq \delta_{k}
$$

Since $\mathcal{H}^{s}(K), \mathcal{H}^{s}\left(K^{\prime}\right)>0$, by Corollary 2.14 of [11], for $\mathcal{H}^{s}$-almost all $x \in K$, we have

$$
\lim _{r \rightarrow 0} \frac{\mathcal{H}^{s}(B(x, r) \cap K)}{\mathcal{H}^{s}(B(x, r) \cap F)}=\lim _{r \rightarrow 0} \frac{\mathcal{H}^{s}\left(B(f(x), r) \cap K^{\prime}\right)}{\mathcal{H}^{s}\left(B(f(x), r) \cap F^{\prime}\right)}=1 .
$$

Furthermore, considering the point $x$ such that $D(f)(x)$ is close to $a$, we can take points $x_{k} \in K$ and $x_{k}^{\prime} \in K^{\prime}$ such that $f\left(x_{k}\right)=x_{k}^{\prime}$ and

$$
\begin{aligned}
1-\frac{\mathcal{H}^{s}\left(B\left(x_{k}, r_{k}\right) \cap K\right)}{\mathcal{H}^{s}\left(B\left(x_{k}, r_{k}\right) \cap F\right)} & \leq \varsigma_{k}, \\
1-\frac{\mathcal{H}^{s}\left(B\left(x_{k}^{\prime},\left(C+2 \kappa^{-1}\right) r_{k}\right) \cap K^{\prime}\right)}{\mathcal{H}^{s}\left(B\left(x_{k}^{\prime},\left(C+2 \kappa^{-1}\right) r_{k}\right) \cap F^{\prime}\right)} & \leq \varsigma_{k}, \\
a-\frac{\mathcal{H}^{s}\left(f\left(B\left(x_{k}, r_{k}\right) \cap K\right)\right)}{\mathcal{H}^{s}\left(B\left(x_{k}, r_{k}\right) \cap K\right)} & \leq \varepsilon_{k} .
\end{aligned}
$$

We can take a largest fine copy $F_{\mathbf{i}_{k}}$ such that

$$
x_{k} \in F_{\mathbf{i}_{k}} \subset B\left(x_{k}, r_{k}\right) \text {. }
$$


Then $\left|F_{\mathbf{i}_{k}}\right| \geq c_{1} r_{k}$ with $c_{1}=\min _{i} r_{i}$. Since $\{1, \cdots, m\}^{N}$ contains $\left(2^{\left(m^{N}\right)}-1\right)$ nonempty subsets, we have the following result.

Lemma 8. There is an integer $M_{k} \leq 2^{\left(m^{N}\right)}-1$ such that for any word $u_{1} \cdots u_{M_{k}+1}$ with length $M_{k}+1$, there exists word $v_{1} \cdots v_{t}$ with $0 \leq t \leq M_{k}$ satisfying

$$
\bar{\Omega}_{\mathbf{i}_{k} *\left(u_{1} \cdots u_{M_{k}+1}\right)}=\bar{\Omega}_{\mathbf{i}_{k} *\left(v_{1} \cdots v_{t}\right)},
$$

Proof. Fix $k$. For any $M \geq 1$, we consider the set

$$
\Xi_{M}=\cup_{l=1}^{M}\left\{\bar{\Omega}_{\mathbf{i}_{k} *\left(u_{1} \cdots u_{l}\right)}: u_{1} \cdots u_{l} \in\{1, \cdots, n\}^{l}\right\} .
$$

Then we have $\operatorname{card}\left(\Xi_{M}\right) \leq 2^{\left(m^{N}\right)}-1$ and

$$
\Xi_{1} \subset \Xi_{2} \subset \cdots \subset \Xi_{M-1} \subset \Xi_{M} \subset \cdots \subset \Xi_{2^{\left(m^{N}\right)}-1} \subset \Xi_{2^{\left(m^{N}\right)}} .
$$

If $\Xi_{M}=\Xi_{M+1}$ for some $M \leq 2^{\left(m^{N}\right)}-1$, then $M$ is the integer desired.

Otherwise, for any $M \leq 2^{\left(m^{\bar{N}}\right)}-1$,

$$
\operatorname{card}\left(\Xi_{M+1}\right)-\operatorname{card}\left(\Xi_{M}\right) \geq 1,
$$

which implies

$$
\begin{aligned}
2^{\left(m^{N}\right)}-1 & \geq \operatorname{card}\left(\Xi_{2^{\left(m^{N}\right)}}\right) \\
& \geq \operatorname{card}\left(\Xi_{1}\right)+\sum_{i=1}^{2^{\left(m^{N}\right)}-1}\left[\operatorname{card}\left(\Xi_{M+1}\right)-\operatorname{card}\left(\Xi_{M}\right)\right] \\
& \geq 1+\left(2^{\left(m^{N}\right)}-1\right)=2^{\left(m^{N}\right)} .
\end{aligned}
$$

This is a contradiction.

Since $M_{k} \leq 2^{\left(m^{N}\right)}-1$, for any word $u_{1} \cdots u_{s}$ with $0 \leq s \leq M_{k}+1\left(\leq 2^{\left(m^{N}\right)}\right)$, we have

$$
\left|F_{\mathbf{i}_{k} *\left(u_{1} \cdots u_{s}\right)}\right| \geq\left|F_{\mathbf{i}_{k}}\right|\left(\min _{i} r_{i}\right)^{2^{\left(m^{N}\right)}} \geq r_{k}\left(\min _{i} r_{i}\right)^{2^{\left(m^{N}\right)}+1} .
$$

Therefore, for any $u_{1} \cdots u_{s}$ with $s \leq M_{k}+1\left(\leq 2^{\left(m^{N}\right)}\right)$,

$$
\left|F_{\mathbf{i}_{k} *\left(u_{1} \cdots u_{s}\right)}\right| \geq c r_{k} \text { and } F_{\mathbf{i}_{k} *\left(u_{1} \cdots u_{s}\right)} \subset B\left(x_{k}, r_{k}\right),
$$

where $c=\left(\min _{i} r_{i}\right)^{2^{\left(m^{N}\right)}+1}$ defined as above. For any word $\mathbf{j}$ with length $|\mathbf{j}|=$ $\left(M_{k}+1\right)$, there exists $\mathbf{j}^{\prime}$ with length $\left|\mathbf{j}^{\prime}\right| \leq M_{k}$ such that

$$
\bar{\Omega}_{\mathbf{i}_{k} * \mathbf{j}}=\bar{\Omega}_{\mathbf{i}_{k} * \mathbf{j}^{\prime}} .
$$

By Lemma 4, Remark 6 and (5.7), the following sets are finite:

$$
\begin{gathered}
\left\{\bar{\Omega}_{\mathbf{i}_{k} *\left(u_{1} \cdots u_{s}\right)}: k \geq 1, u_{1} \cdots u_{s} \in\{1, \cdots, m\}^{s} \text { with } s \leq M_{k}+1\right\}, \\
\left\{\bar{\beta}\left(\mathbf{i}_{k} *\left(u_{1} \cdots u_{s}\right), u\right): k \geq 1, u_{1} \cdots u_{s} \in\{1, \cdots, m\}^{s} \text { with } s \leq M_{k}, 1 \leq u \leq n\right\}, \\
\left\{\bar{\Delta}_{\mathbf{i}_{k} *\left(u_{1} \cdots u_{s}\right), u}: k \geq 1, u_{1} \cdots u_{s} \in\{1, \cdots, m\}^{s} \text { with } s \leq M_{k}, 1 \leq u \leq n\right\} .
\end{gathered}
$$


Therefore, we can select a subsequence $k_{1}<k_{2}<\cdots<k_{t}<\cdots$ with $\lim _{t \rightarrow \infty} k_{t}=$ $\infty$ such that for all $t$,

$$
\begin{aligned}
M_{k_{t}} & =M_{k_{t+1}} \equiv M, \\
\bar{\Omega}_{\mathbf{i}_{k_{t}} *\left(u_{1} \cdots u_{s}\right)} & =\bar{\Omega}_{\mathbf{i}_{k_{t+1}} *\left(u_{1} \cdots u_{s}\right)}, \\
\bar{\Delta}_{\mathbf{i}_{k_{t}} *\left(u_{1} \cdots u_{s}\right), u} & =\bar{\Delta}_{\mathbf{i}_{k_{t+1}} *\left(u_{1} \cdots u_{s}\right), u,}, \\
\bar{\beta}\left(\mathbf{i}_{k_{t}} *\left(u_{1} \cdots u_{s}\right), u\right) & =\bar{\beta}\left(\mathbf{i}_{k_{t+1}} *\left(u_{1} \cdots u_{s}\right), u\right),
\end{aligned}
$$

for any word $u_{1} \cdots u_{s}$ with $0 \leq s \leq M$ and any $u$ with $1 \leq u \leq n$. Denote

$$
\begin{aligned}
\Omega_{u_{1} \cdots u_{s}} & =\bar{\Omega}_{\mathbf{i}_{k_{t}} *\left(u_{1} \cdots u_{s}\right)}, \\
\Delta_{u_{1} \cdots u_{s}, u} & =\bar{\Delta}_{\mathbf{i}_{k_{t}} *\left(u_{1} \cdots u_{s}\right), u}, \\
\beta\left(\left(u_{1} \cdots u_{s}\right), u\right) & =\bar{\beta}\left(\mathbf{i}_{k_{t}} *\left(u_{1} \cdots u_{s}\right), u\right) .
\end{aligned}
$$

Then

Notice that

$$
\Omega_{u_{1} \cdots u_{s}}=\cup_{u=1}^{n} \Delta_{u_{1} \cdots u_{s}, u}
$$

$$
\Delta_{u_{1} \cdots u_{s}, u_{s+1}} \prec \Omega_{u_{1} \cdots u_{s} u_{s+1}} \text { for } 0 \leq s \leq M-1 .
$$

For word $u_{1} \cdots u_{M} u_{M+1}$, there exists $v_{1} \cdots v_{s}$ with $0 \leq s \leq M$ such that

$$
\Delta_{u_{1} \cdots u_{M}, u_{M+1}}=\beta\left(u_{1} \cdots u_{M}, u_{M+1}\right) \Omega_{v_{1} \cdots v_{s}}
$$

that is

$$
\Delta_{u_{1} \cdots u_{M}}, u_{M+1} \prec \Omega_{v_{1} \cdots v_{s}} .
$$

According to Lemma 3 , we shall verify that for all $u$ and $u_{1} \cdots u_{s}$ with $s \leq M$,

$$
\frac{v\left(\Delta_{u_{1} \cdots u_{s}, u}\right)}{v\left(\Omega_{u_{1} \cdots u_{s}}\right)}=r_{u}^{s}
$$

where $v$ is defined in Subsection 5.1 .

Let $\mathbf{i}=\mathbf{i}_{k_{t}} *\left(u_{1} \cdots u_{s}\right)$. Then it follows from (5.28) and Lemma 5 that

$$
\frac{\mathcal{H}^{s}\left(f\left(F_{\mathbf{i} * u} \cap K\right)\right)}{\mathcal{H}^{s}\left(F_{\mathbf{i} * u} \cap K\right)}, \frac{\mathcal{H}^{s}\left(f\left(F_{\mathbf{i}} \cap K\right)\right)}{\mathcal{H}^{s}\left(F_{\mathbf{i}} \cap K\right)} \geq a-c^{*}\left[1-c^{*} \varsigma_{k_{t}}\right]^{-1} \varepsilon_{k_{t}} .
$$

By (5.27), we have

$$
\left|\left[\frac{\mathcal{H}^{s}\left(f\left(F_{\mathbf{i}} \cap K\right)\right)}{\mathcal{H}^{s}\left(F_{\mathbf{i}} \cap K\right)}\right] /\left[\frac{\mathcal{H}^{s}\left(f\left(F_{\mathbf{i} * u} \cap K\right)\right)}{\mathcal{H}^{s}\left(F_{\mathbf{i} * u} \cap K\right)}\right]-1\right| \leq \delta_{k_{t}}
$$

That means

$$
\left|\left[\frac{\mathcal{H}^{s}\left(F_{\mathbf{i} * u} \cap K\right)}{\mathcal{H}^{s}\left(F_{\mathbf{i}} \cap K\right)}\right] /\left[\frac{\mathcal{H}^{s}\left(f\left(F_{\mathbf{i} * u} \cap K\right)\right)}{\mathcal{H}^{s}\left(f\left(F_{\mathbf{i}} \cap K\right)\right)}\right]-1\right| \leq \delta_{k_{t}} .
$$

It follows from (5.28) and Lemma 5 ,

$$
\frac{\mathcal{H}^{s}\left(F_{\mathbf{i}} \cap K\right)}{\mathcal{H}^{s}\left(F_{\mathbf{i}}\right)}, \frac{\mathcal{H}^{s}\left(F_{\mathbf{i} * u} \cap K\right)}{\mathcal{H}^{s}\left(F_{\mathbf{i} * u}\right)} \geq 1-c^{*} \varsigma_{k_{t}} .
$$

Therefore,

$$
\left|\left[\frac{\mathcal{H}^{s}\left(F_{\mathbf{i} * u} \cap K\right)}{\mathcal{H}^{s}\left(F_{\mathbf{i}} \cap K\right)}\right] /\left[\frac{\mathcal{H}^{s}\left(F_{\mathbf{i} * u}\right)}{\mathcal{H}^{s}\left(F_{\mathbf{i}}\right)}\right]-1\right| \leq \frac{1}{1-c^{*} \varsigma_{k_{t}}}-1 .
$$

On the other hand,

$$
\frac{\mathcal{H}^{s}\left(F_{\mathbf{i} * u}\right)}{\mathcal{H}^{s}\left(F_{\mathbf{i}}\right)}=r_{u}^{s}
$$


Suppose $F_{\mathbf{j}(\mathbf{i})}^{\prime}$ is the smallest fine copy containing $f\left(F_{\mathbf{i}} \cap K\right)$, and

$$
\bigcup_{j^{*} \in \Lambda_{\mathbf{i}}}\left(F_{\mathbf{j}(\mathbf{i}) * j^{*}}^{\prime} \cap K^{\prime}\right)=\cup_{u=1}^{n}\left[\bigcup_{v^{*} \in \Lambda_{\mathbf{i} * u}} F_{\mathbf{j}(\mathbf{i}) * \bar{\beta}(\mathbf{i}, u) * v^{*}}^{\prime} \cap K^{\prime}\right] .
$$

In fact, for $\mathbf{i}=\mathbf{i}_{k_{t}} *\left(u_{1} \cdots u_{s}\right)$, we have

$$
\frac{v\left(\Delta_{u_{1} \cdots u_{s}, u}\right)}{v\left(\Omega_{u_{1} \cdots u_{s}}\right)}=\frac{\sum_{v^{*} \in \Lambda_{\mathbf{i} * u}} \mathcal{H}^{s}\left(F_{\mathbf{j}(\mathbf{i}) * \bar{\beta}(\mathbf{i}, u) * v^{*}}^{\prime}\right)}{\sum_{j^{*} \in \Lambda_{\mathbf{i}}} \mathcal{H}^{s}\left(F_{\mathbf{j}(\mathbf{i}) * j^{*}}^{\prime}\right)} .
$$

Applying (5.21), (5.22), (5.26) to Lemmas 6, we obtain a constant $d^{*}$ such that for $j^{*} \in \Lambda_{\mathbf{i}}$ and $v^{*} \in \Lambda_{\mathbf{i} * u}$,

$$
\begin{aligned}
& \max \left(1-\frac{\mathcal{H}^{s}\left(F_{\mathbf{j}(\mathbf{i}) * j^{*}}^{\prime} \cap K\right)}{\mathcal{H}^{s}\left(F_{\mathbf{j}(\mathbf{i}) * j^{*}}^{\prime}\right)}, 1-\frac{\mathcal{H}^{s}\left(F_{\mathbf{j}(\mathbf{i}) * \bar{\beta}(\mathbf{i}, u) * v^{*}}^{\prime} \cap K\right)}{\mathcal{H}^{s}\left(F_{\mathbf{j}(\mathbf{i}) * \bar{\beta}(\mathbf{i}, u) * v^{*}}^{\prime}\right)}\right) \\
\leq & d^{*}\left[1-\frac{\mathcal{H}^{s}\left(B\left(x_{k_{t}}^{\prime},\left(C+2 \kappa^{-1}\right) r_{k_{t}}\right) \cap K^{\prime}\right)}{\mathcal{H}^{s}\left(B\left(x_{k_{t}}^{\prime},\left(C+2 \kappa^{-1}\right) r_{k_{t}}\right) \cap F^{\prime}\right)}\right] \\
\leq & d^{*} \varsigma_{k_{t}} .
\end{aligned}
$$

For $\mathbf{i}=\mathbf{i}_{k_{t}} *\left(u_{1} \cdots u_{s}\right)$, by (5.38) and (5.39), we have

$$
\begin{aligned}
& \left|\left[\frac{\mathcal{H}^{s}\left(f\left(F_{\mathbf{i} * u} \cap K\right)\right)}{\mathcal{H}^{s}\left(f\left(F_{\mathbf{i}} \cap K\right)\right)}\right] /\left[\frac{v\left(\Delta_{u_{1} \cdots u_{s}, u}\right)}{v\left(\Omega_{u_{1} \cdots u_{s}}\right)}\right]-1\right| \\
= & \left|\left[\frac{\sum_{v^{*} \in \Lambda_{\mathbf{i} * u}} \mathcal{H}^{s}\left(F_{\mathbf{j}(\mathbf{i}) * \bar{\beta}(\mathbf{i}, u) * v^{*}}^{\prime} \cap K\right)}{\sum_{v^{*} \in \Lambda_{\mathbf{i} * u}} \mathcal{H}^{s}\left(F_{\mathbf{j}(\mathbf{i}) * \bar{\beta}(\mathbf{i}, u) * v^{*}}^{\prime}\right)}\right] /\left[\frac{\sum_{j^{*} \in \Lambda_{\mathbf{i}}} \mathcal{H}^{s}\left(F_{\mathbf{j}(\mathbf{i}) * j^{*}}^{\prime} \cap K\right)}{\sum_{j^{*} \in \Lambda_{\mathbf{i}}} \mathcal{H}^{s}\left(F_{\mathbf{j}(\mathbf{i}) * j^{*}}^{\prime}\right)}\right]-1\right| \\
\leq & \frac{1}{1-d^{*} \varsigma_{k_{t}}}-1 .
\end{aligned}
$$

Letting $t \rightarrow \infty$, we get (5.33) from (5.35), (5.36), (5.37) and (5.40).

Finally, Theorem 3 follows from (5.30)-(5.33) and Lemma 3.

\section{Bilipschitz Embedding about 1-Regular Set}

Given $s$-regular set $E$ and $t$-regular set $F$ with $s<t$, [12] proved that for any $s_{1}<s$, there is a $s_{1}$-regular subset of $E$, which can be bilipschitz embedded into $F$. How about $s_{1}=s$ ? Investigate the following question:

Can we find a subset of $E$ with positive s-dimensional Hausdorff measure, such that $E$ can be bilipschitz embedded into $F$ ?

In fact, this is true for $s<1$ ([12]), but this is false for $s=1$ by Proposition 4 .

Proposition 4. Suppose $B$ is a subset of $[0,1]$ with positive Lebesgue measure. Then $B$ can not be bilipschitz embedded into any self-similar set satisfying SSC.

Proof. Suppose there is a bilipschitz mapping $f: B \rightarrow f(B) \subset E$, where $E$ is a self-similar set satisfying SSC. Let $C$ be the bilipschitz constant of $f$. Denote by $\mathcal{L}$ the Lebesgue measure on $\mathbb{R}^{1}$.

Assume $x \in B$ is a Lebesgue point, i.e.,

$$
\mathcal{L}[(x-\varepsilon, x+\varepsilon) \cap B] / 2 \varepsilon \rightarrow 1, \text { as } \varepsilon \rightarrow 0,
$$

which implies that

$$
\mathcal{L}[(x-\varepsilon, x+\varepsilon) \backslash B] / 2 \varepsilon \rightarrow 0, \text { as } \varepsilon \rightarrow 0 .
$$


Suppose

$$
\mathcal{L}[(x-\varepsilon, x+\varepsilon) \backslash B] / 2 \varepsilon<\varsigma .
$$

Let $E_{i_{1} \cdots i_{k}}$ be the smallest fine copy of $E$ which contains $f((x-\varepsilon, x+\varepsilon) \cap B)$. Hence

$$
\begin{aligned}
\left|E_{i_{1} \cdots i_{k}}\right| & \geq|f((x-\varepsilon, x+\varepsilon) \cap B)| \\
& \geq C^{-1}|(x-\varepsilon, x+\varepsilon) \cap B| \\
& \geq C^{-1} 2 \varepsilon(1-\varsigma) .
\end{aligned}
$$

There are two distinct points

$$
f(x), f(y) \in E \text { with } x, y \in(x-\varepsilon, x+\varepsilon) \cap B,
$$

such that

$$
\begin{aligned}
& f(x) \in f[(x-\varepsilon, x+\varepsilon) \cap B)] \cap E_{i_{1} \cdots i_{k} i_{k+1}}, \\
& f(y) \in f[(x-\varepsilon, x+\varepsilon) \cap B)] \cap E_{i_{1} \cdots i_{k} i_{k+1}^{\prime}},
\end{aligned}
$$

with $i_{k+1} \neq i_{k+1}^{\prime}$.

However, since $x, y \in(x-\varepsilon, x+\varepsilon) \cap B$, there is a sequence of points in $B$,

$$
(x=) z_{0}, z_{1}, \cdots, z_{k-1}, z_{k}(=y) \in B
$$

such that for every $i$,

$$
\left|z_{i}-z_{i+1}\right| \leq 2 \varsigma \varepsilon
$$

Then there is a sequence of points in $f(B)$,

$$
(f(x)=) f\left(z_{0}\right), f\left(z_{1}\right), \cdots, f\left(z_{k-1}\right), f\left(z_{k}\right)(=f(y)) \in B,
$$

such that for every $i$,

$$
\left|f\left(z_{i}\right)-f\left(z_{i+1}\right)\right| \leq(2 C \varepsilon) \varsigma .
$$

Since $i_{k+1} \neq i_{k+1}^{\prime}$, there is an index $i^{*}$ such that $f\left(z_{i^{*}}\right), f\left(z_{i^{*}+1}\right)$ belong to the different pieces in $\left\{E_{i_{1} \cdots i_{k}}\right\}_{j}$.

Let $\left\{r_{i}\right\}_{i}$ be the ratios set of $E$. Then we have

$$
\begin{aligned}
& \left|f\left(z_{i^{*}}\right)-f\left(z_{i^{*}+1}\right)\right| \\
\geq & \min _{j \neq j^{\prime}} d\left(E_{i_{1} \cdots i_{k} j}, E_{i_{1} \cdots i_{k} j^{\prime}}\right) \\
\geq & \left(\min _{i} r_{i}\right)\left|E_{i_{1} \cdots i_{k}}\right| \\
\geq & \left(\min _{i} r_{i}\right) C^{-1}(2 \varepsilon(1-\varsigma)),
\end{aligned}
$$

which contradicts (6.1) when $\varsigma$ is taken small enough.

Example 3. Let $E=[0,1] \times\{0\}$ is a self-similar set of $\mathbb{R}^{2}$ generated by

$$
T_{1}(x, y)=(x, y) / 3, T_{2}(x, y)=(x, y) / 3+(1 / 3,0), T_{3}(x, y)=(x, y) / 3+(2 / 3,0) .
$$

Let $E^{\prime}$ be a self-similar set of $\mathbb{R}^{2}$ generated by

$$
T_{1}^{\prime}(x, y)=(x, y) / 3, T_{2}^{\prime}(x, y)=(x, y) / 3+(2 / 3,0), T_{3}^{\prime}(x, y)=(x, y) / 3+(0,2 / 3) .
$$

Then $E^{\prime}$ satisfies SSC. By Proposition 4 , any subset of $E$ with positive $\mathcal{H}^{1}$ measure cannot be bilipschitz embedded into $F$. 


\section{REFERENCES}

[1] M. Bonk, Quasiconformal geometry of fractals. International Congress of Mathematicians. Vol. II, 1349-1373, Eur. Math. Soc., Zürich, 2006.

[2] D. Cooper and T. Pignataro. On the shape of Cantor sets. J. Differential Geom., 28(2):203$221,1988$.

[3] G. David and S. Semmes. Fractured fractals and broken dreams, volume 7 of Oxford Lecture Series in Mathematics and its Applications. The Clarendon Press Oxford University Press, New York, 1997. Self-similar geometry through metric and measure.

[4] K. J. Falconer. Techniques in fractal geometry. John Wiley \& Sons, Ltd., Chichester, 1997.

[5] K. J. Falconer and D. T. Marsh. Classification of quasi-circles by Hausdorff dimension. Nonlinearity, 2(3):489-493, 1989.

[6] K. J. Falconer and D. T. Marsh. On the Lipschitz equivalence of Cantor sets. Mathematika, 39(2):223-233, 1992.

[7] B. Farb, L. Mosher, A rigidity theorem for the solvable Baumslag-Solitar groups. With an appendix by Daryl Cooper. Invent. Math. 131 (1998), no. 2, 419-451.

[8] D. Feng, Z. Y. Wen, and J. Wu. Some dimensional results for homogeneous Moran sets. Sci. China Ser. A, 40(5):475-482, 1997.

[9] J.E. Hutchinson, Fractals and self similarity, Indiana Univ. Math. J., 30:713-747, 1981.

[10] R. Kenyon, Projecting the one-dimensional Sierpinski gasket, Israel J. Math. 97:221-238, 1997.

[11] P. Mattila, Geometry of Sets and Measures in Euclidean Spaces, Cambridge University Press, 1995.

[12] P. Mattila and P. Saaranen. Ahlfors-David regular sets and bilipschitz maps, arXiv.org, preprint.

[13] P. A. P. Moran. Additive functions of intervals and Hausdorff measure. Proc. Cambridge Philos. Soc., 42:15-23, 1946.

[14] R.D.Mauldin and S.C.Williams, Hausdorff dimension in graph directed constructions, Trans. Amer. Math. Soc. 309(1-2): 811-839, 1988.

[15] H. Rao, H. J. Ruan, and L. F. Xi. Lipschitz equivalence of self-similar sets. C. R. Math. Acad. Sci. Paris, 342(3):191-196, 2006.

[16] H. J. Ruan and L. F. Xi, Lipschitz equivalence of self-similar sets with touching structure, submitted.

[17] H. Rao, Z. Y. Wen, and J. Wu. The net measure property of Moran sets and its applications. Kexue Tongbao (Chinese), 42(22):2383-2387, 1997.

[18] Z. Y. Wen and L. F. Xi. Relations among Whitney sets, self-similar arcs and quasi-arcs. Israel J. Math., 136:251-267, 2003.

[19] L. F. Xi. Lipschitz equivalence of self-conformal sets. J. London Math. Soc. (2), 70(2):369$382,2004$.

[20] L. F. Xi. Quasi-Lipschitz equivalence of fractals. Israel J. Math., 160:1-21, 2007.

[21] L. F. Xi. Lipschitz equivalence of dust-like self-similar sets, Math. Z., Math. Z., published online in 2009.

[22] L. F. Xi and H. J. Ruan. Lipschitz equivalence of generalized $\{1,3,5\}-,\{1,4,5\}$ self-similar sets. Sci. China Ser. A, 50(11):1537-1551, 2007.

[23] L. F. Xi, H. J. Ruan, and Q. L. Guo. Sliding of self-similar sets. Sci. China Ser. A, 50(3):351360, 2007.

[24] L. F. Xi and Y. Xiong, Lipschitz equivalence of graph-directed fractals, Studia Math., Studia Math., 194(2009):197-205.

[25] L. F. Xi and Y. Xiong, Self-similar sets with initial cubic patterns, submitted. 
Department of Mathematics, Tsinghua University, Beijing, 100084, P. R. China

E-mail address: dengj07@mails.tsinghua.edu.cn

Department of Mathematics, Tsinghua University, Beijing, 100084, P. R. China

E-mail address: wenzy@mail.tsinghua.edu.cn

Department of Mathematics, South China University of Technology, Guangzhou, 510641, P. R. China

E-mail address: xiongyng@gmail.com

Institute of Mathematics, Zhejiang Wanli University, Ningbo, Zhejiang, 315100, P. R. CHINA

E-mail address: xilifengningbo@yahoo.com 